\title{
9. MIOCENE DISSOLUTION FACIES AND MICROFACIES AT NORTHWEST ATLANTIC DEEP SEA DRILLING PROJECT SITE $603^{1}$
}

\author{
Elisabeth Gervais, Instituut voor Aardwetenschappen, Vrije Universiteit, Amsterdam²
}

\begin{abstract}
Depth fluctuations of the lysocline and calcite compensation depth $(\mathrm{CCD})$ through time were investigated at Deep Sea Drilling Project Site 603, Leg 93. The CCD fell during the middle Miocene at the onset of the Western Boundary Undercurrent, correlated with seismic Horizon X. Subsequently deposited units show fluctuations of the dissolution curve. Major changes in dissolution facies correspond with lithologic boundaries.
\end{abstract}

\section{INTRODUCTION}

Middle Miocene to early Pliocene sediment samples from DSDP Site 603, Leg 93 (Fig. 1) consist of hemipelagic clay and claystone (Blake Ridge Formation) and contain calcareous foraminifers. The preservation of these foraminifers decreases downward in the section, and below the middle Miocene sediments no carbonates are present. Using the calcite compensation depth (CCD) curve of Tucholke and Vogt (1979, p. 796, fig. 3), which is based on the back-tracking of several DSDP sites, and adding half the sediment fill to the present water depth $(4633 \mathrm{~m})$, Site 603 should have risen above the CCD in the middle Miocene.

The objective of this study is to investigate the nature and rate of the change from a depositional environment below the $\mathrm{CCD}$ to one above the lysocline, through the transitional environment between the $\mathrm{CCD}$ and the lysocline.

The dissolution of foraminifers has been successfully used to indicate depth changes of the foraminiferal lysocline and CCD. Berger and von Rad (1972) recognized several stages of dissolution ("dissolution facies") based on calcareous planktonic/benthic foraminifer ratio, percentage of foraminiferal fragments, percentage of dissolution-resistant foraminifers, and diversity; they gave each stage a code ("FS number"), as specified in Table 1. FS number 3 roughly equates to lysocline depth; all higher numbers signify a dissolution facies between the lysocline and the CCD. Berger (1979) gave an overview of the principles and applications of this method.

This FS measure is used in this study of the foraminiferal lysocline and CCD movements at DSDP Site 603, Leg 93.

\section{METHODS}

Berger and von Rad (1972) give 10 states of preservation (FS numbers) for pelagic sediments (Table 1). The dissolution facies of all samples were determined by counting the whole and/or fragmented plank-

\footnotetext{
1 van Hinte, J. E., Wise, S. W., Jr., et al., Init. Repts. DSDP, 93: Washington (U.S. Govt, Printing Office).

2 Address: Instituut voor Aardwetenschappen, Vrije Universiteit, P.O. Box 7161, 1007 MC Amsterdam, The Netherlands.
}

tonic and benthic foraminifers; estimating the percentage of foraminiferal fragments in a representative split of the residue; and then calculating the percentage of planktonic to total foraminiferal fauna and the percentage of resistant planktonic foraminiferal forms (Table 2). It is noted that FS numbers are influenced by introduction of displaced (possibly contemporaneous) and reworked (certainly older) foraminifers; generally these factors produce lower FS numbers. Displaced sediments are defined by washed residues containing $\geq 3 \%$ quartz, Quinqueloculina sp., reworked Cretaceous foraminifers, $\geq 95 \%$ rock fragments (predominantly silt), echinoid, ostracode, and shell fragments, and glauconite, or a combination of these components. The percentages of foraminifers were calculated from the fraction between 125 and $250 \mu \mathrm{m}$.

A total of 161 samples was analyzed. To prevent fragmentation of calcareous foraminifers in sample preparation, the samples were soaked in a soda solution until the sediments (clays) disintegrated and then were carefully washed in a weak stream of water. More intensely lithified samples of Core 25 and Cores 42 to 54 (with the exception of Samples $603-42-1,30-34 \mathrm{~cm}$ and $603-46-1,30-34 \mathrm{~cm}$ ) required soaking in both a soda and a $5 \%$ peroxide solution prior to washing.

Table 3 shows estimated percentages of the washed residue components of the core samples. The solution susceptibility list of planktonic foraminifers given by Hemleben and Auras (1984) was used (Table 4), and species with a solution susceptibility $\geq 5$ are considered resistant. Similar to findings of Hemleben and Auras (1984) on Miocene and Pliocene material from the Central Atlantic, Globigerinoides conglobatus has undergone secondary calcification in all the Hole 603 samples and has therefore become more resistant to dissolution.

\section{RESULTS}

The sediment of Cores 54 to 46 was deposited below the $C C D$, and the sample residues only contain sporadic agglutinated foraminifers and large amounts of silt $(\geq 95 \%)$. In and above Core 45 , the dissolution curve fluctuates between the CCD and the lysocline (FS numbers 3-10, Fig. 2). The first minor fluctuations are present between Cores 45 and 42 . In sediment samples between Cores 41 and 36 (with the exception of reworked sediments of Samples 603-41-3, 30-34 cm and 603-41-6, $30-34 \mathrm{~cm}$ ), absence of calcareous foraminifers and their fragments points to a return to a depositional environment below the CCD.

The first substantial fluctuations between the CCD and the lysocline are present between Cores 36 and 32, followed by an interval of minor fluctuations between Cores 31 and 25. Samples 603-25-1, 30-32 cm; 603-25-2, $30-32 \mathrm{~cm}$, and 603-25-6, 30-32 cm contain reworked Cretaceous foraminifers (Table 2). A second interval of substantial fluctuations has been found in Core 24 to the 


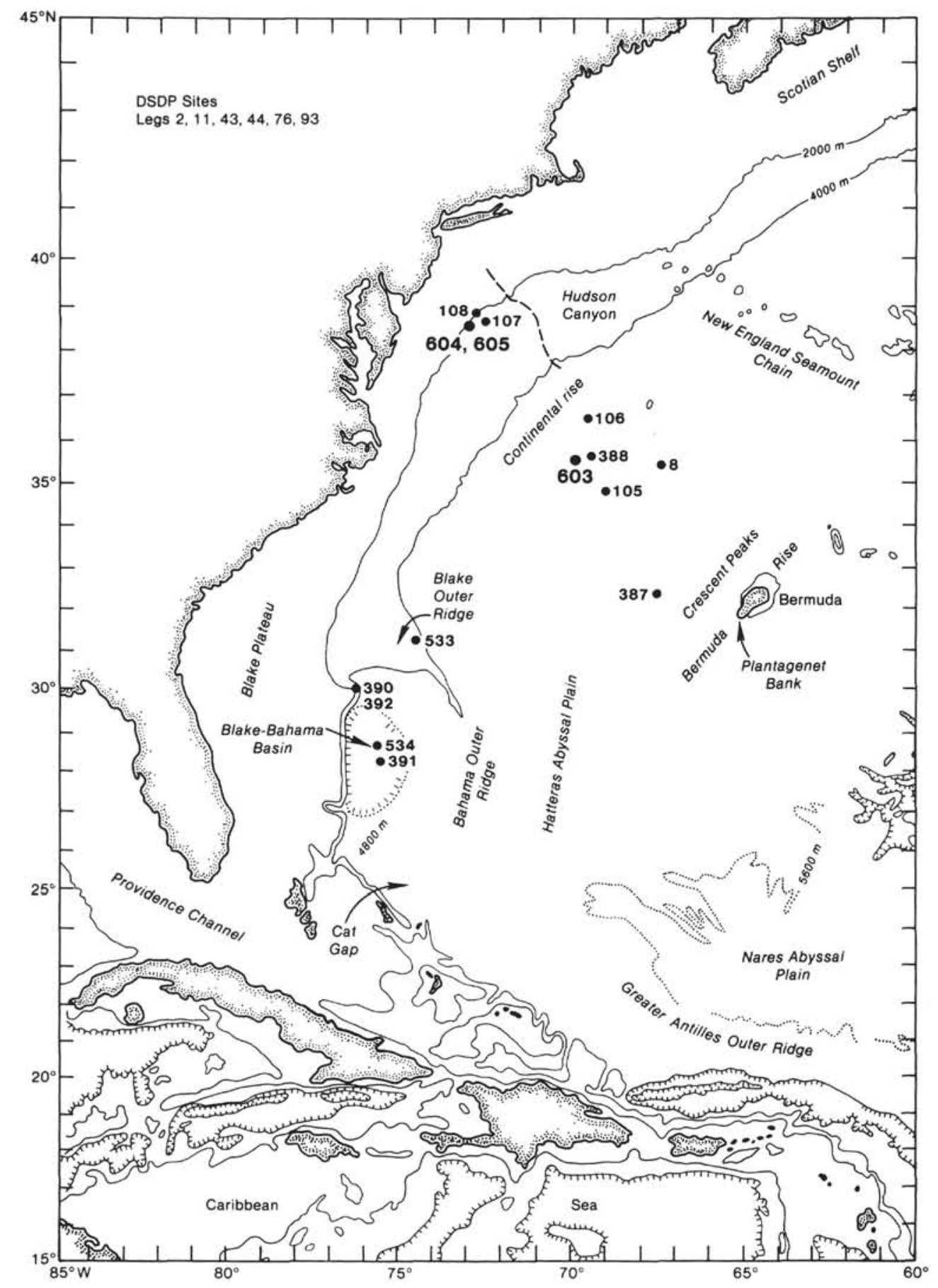

Figure 1. Main physiographic features of the northwestern Atlantic and location of DSDP Sites from Legs 2, 11, $43,44,76$, and 93 . DSDP Site 603 of Leg 93 was drilled on the lower continental rise.

lower part of Core 22, followed by minor fluctuations in the upper part of Core 22 to Cores 21 and 20 .

In the intervals with minor fluctuations, a few samples contain flattened foraminifers (Samples 603-22-6, $30-34 \mathrm{~cm}$; 603-26-5, 32-36 cm; 603-29-3, 30-34 cm; $603-29-4,30-34 \mathrm{~cm}$, and 603-31-1, 37-39 cm). The fauna of Cores 19 to 13 commonly contains displaced elements; these sediments have been deposited above the CCD, with the exception of Sample 603-17-2, 30-34 cm.
All residues of Core 16 and Sample 603-17-1, 30-34 cm contain little or no rock fragments and no bassanite (sulfate), in contrast to those above and below these samples. Samples 603-18-1, 30-34 cm, 603-19-2, 30-34 cm, 603-19-5, 30-34 cm and 603-19-6, 30-34 cm, contain reworked Cretaceous foraminifers: Globigerinelloides sp., Heterohelix sp., and Globotruncana sp.

The relatively low percentages of resistant planktonic foraminifers in Cores 17 to 13 is due to a decrease in dis- 
Table 1. Calcium carbonate solution facies based on calcareous foraminifers, from Berger and von Rad (1972).

\begin{tabular}{|c|c|c|}
\hline $\begin{array}{c}\text { FS } \\
\text { number }\end{array}$ & Criteria & $\begin{array}{c}\text { Approximate } \\
\text { loss } \\
(\%)\end{array}$ \\
\hline 1 & $\begin{array}{l}\text { Foraminifers undissolved (aragonitic pteropods } \\
\text { present) }\end{array}$ & 0 \\
\hline 2 & $\begin{array}{l}\text { Hastigerina present; many globigerinids bear } \\
\text { spines; } \mathrm{R}<5 \%\end{array}$ & $\sim 10$ \\
\hline 3 & $\begin{array}{l}\text { More spined than nonspined species; } 5 \%<\mathrm{R} \\
<25 \%\end{array}$ & $<50$ \\
\hline 4 & $\begin{array}{l}\text { Maximum equitability of assemblage; } 25 \%<\mathrm{R} \\
<50 \%\end{array}$ & $50-80$ \\
\hline 5 & $\begin{array}{l}\text { Solution obvious; whole tests }>\text { fragments; } \\
\qquad R>50 \%\end{array}$ & $80-90$ \\
\hline 6 & $\begin{array}{l}\text { Fragments > whole tests; planktonic whole } \\
\text { tests }>\text { calcareous benthics } \mathrm{f} \text {. }\end{array}$ & $>90$ \\
\hline 7 & $\begin{array}{l}\text { Calcareous benthics > planktonics (planktonics } \\
\quad \neq 0)\end{array}$ & $>95$ \\
\hline 8 & $\begin{array}{l}\text { No whole planktonics; calcareous benthics } \\
\text { present }\end{array}$ & $>98$ \\
\hline 9 & Fragments of calcareous foraminifers only & $>99$ \\
\hline $\mathrm{X}$ & No calcareous fragments & 100 \\
\hline
\end{tabular}

Note: The resistant forms (R) are species with a solution susceptibility $\geq 5$ (rank factors of the planktonic foraminifers are after Hemleben and Auras, 1984). In samples from Cores 603-17 to -13 , the initial $\mathrm{R}$ is smaller because of abundant Globigerina quinqueloba and $G$. juvenilis/glutinata.

solution and the abundance of globigerinids (Globigerina quinqueloba and $G$. glutinata/juvenilis) in the assemblage.

Most of the samples contain the sulfate bassanite $\left(2 \mathrm{CaSO}_{4} \cdot 1 \mathrm{H}_{2} \mathrm{O}\right.$; determined by $\mathrm{X}$-ray analysis) varying from 0.1 to $28.0 \%$ of the wash residue. The bassanite crystals are often encrusted with very small calcite crystals. The large amounts of pyrite (up to $98.5 \%$ in the wash residue), with the availability of organic matter (i.e., plant remains) and sulfate (bassanite), may be the end product of bacterial sulfate reduction.

\section{BIOHORIZONS AND RELATIVE DATING}

Three planktonic foraminiferal biozones are distinguished in Cores 54 to 13 of Hole 603. In terms of the standard zonation of Stainforth et al. (1975), the biozones are: the Globorotalia margaritae Zone, the Neogloboquadrina acostaensis Zone, and the combined Globorotalia siakensis-Globorotalia menardii Zone.

The G. margaritae Zone is based on the first appearance of Globorotalia margaritae Bolli and Bermudez in Core 19, and the absence of Globorotalia crassaformis Galloway and Wissler and the presence of Globigerina nepenthes Todd in Core 13. The base of the N. acostaensis Zone is defined by the first appearance of Neogloboquadrina acostaensis (Blow) in Sample 603-32-2, 94-96 cm and the first appearance of Globorotalia margaritae in Core 19. The Globorotalia siakensis and Globorotalia menardii Zones cannot be separated because of bad preservation or absence of key fossils. Globigerina nepenthes has its first appearance in the middle part of the Globorotalia siakensis Zone (Stainforth et al., 1975 ) and is present (one specimen only) in Core 54 at the bottom of Hole 603, which implies that this core belongs to the G. siakensis Zone. However, Core 54 is contaminated (Site 603 report, this volume), and according to the occurrence of nannofossils, the base of the G. sia- kensis Zone is to be expected between Cores 41 and 51 (Muza et al., this volume). Samples of Core 45 contain more specimens of $G$. nepenthes and are placed in the G. siakensis Zone.

An influx of cold-water fauna marks Sample 603-16-1, 30-34 cm (Globigerina bulloides d'Orbigny, Neogloboquadrina pachyderma [Ehrenberg], and Neogloboquadrina atlantica [Berggren]) and is preceded by the entrance of Globorotalia praemiocenica Lamb and Beard in Sample 603-16-2, 30-34 cm and Globigerinoides conglobatus (Brady) in Sample 603-17-4, 30-34 cm.

\section{DISCUSSION}

The major seismic reflections and important lithologic breaks are conspicuously marked by changes in the dissolution curve (Fig. 2). The Leg 93 scientific party interpreted the seismic facies of the interval from Cores 54 to 46 , which was deposited below the CCD, as distal turbidites. The first fluctuations (Core 45) correspond with Horizon X, which marks the onset of the presentday Western Boundary Undercurrent (WBUC, see Site 603 chapter, this volume). As discussed above, this event happened in the middle Miocene. These first minor fluctuations at the top of lithologic Unit IC (Cores 45-42) end abruptly in Core 41 . This may be the effect of a decrease of the accumulation rate caused by variations of the WBUC contour current and/or a sudden rise in CCD level.

The abrupt end of the relatively low CCD level in the interval between Cores 36 and 32 coincides with seismic reflection Horizon $\mathbf{M}_{2}$. The base of this interval (Core 36) coincides with an unnamed seismic boundary. It is preceded in Core 37 by displaced sediments.

The substantial fluctuations in the interval from Cores 23 and 24 do not seem to have been caused by events that also affected the seismic pattern, or do not show on the seismic record because the zone is too thin and beyond seismic resolution.

Between Cores 20 and 19, near the base of lithologic Unit IA (reflection Horizon M1), calcium carbonate is well preserved. This does not necessarily reflect a drop in CCD level, but can be caused solely by sediment displacements (slumps or mud turbidites), resulting in an influx of shallow-water benthic foraminifers, together with reworked Cretaceous foraminifers (Cores 18 and 19), ostracode and echinoderm fragments, plant remains and glauconite.

The influx of cold-water planktonic foraminifers in Sample 603-16-1, 30-34 cm marks the onset of fair preservation of the autochthonous faunal elements. The calcareous fauna high in the section (Core 16 and above) is generally better preserved (FS $\geq 6$ ). The time interval between the first emergence of the site above the CCD (Core 45 , at approximately $12.0 \mathrm{Ma}$, in Zone M8) to a generally better preserved calcareous facies (Core 16, at approximately $5.4 \mathrm{Ma}$, at the top of Zone M13) is $6.6 \mathrm{~m}$.y. The Neogene geochronologic time scale of Berggren et al. (1985) is used for this calculation.

The Miocene/Pliocene boundary at this site has been drawn above Core 16 and does not coincide with a seismic sequence boundary. In the subtropical to temperate 
Table 2. Data concerning the presence and absence of whole and/or fragmented planktonic and benthic foraminifers, the percentage of foraminiferal fragments, the percentage of planktonic to total foraminiferal fauna, and the percentage of resistant planktonic foraminiferal forms, Hole 603.

\begin{tabular}{|c|c|c|c|c|c|c|c|c|c|c|c|}
\hline $\begin{array}{c}\text { Sample } \\
\text { (Core-Section, } \\
\text { interval in } \mathrm{cm} \text { ) }\end{array}$ & 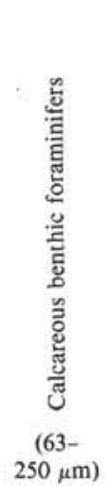 & 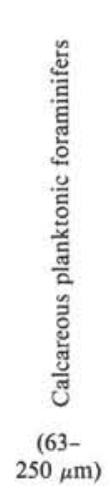 &  & 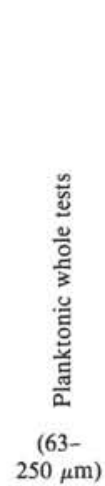 & 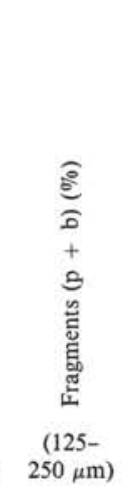 & 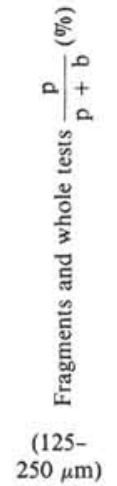 & 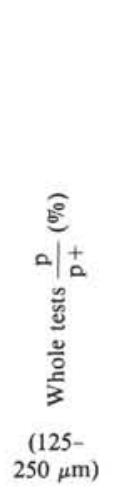 & $\begin{array}{c}\widehat{\varrho} \\
\check{\Xi} \\
(125- \\
250 \mu \mathrm{m})\end{array}$ & $\begin{array}{c}\text { 号 } \\
(63- \\
250 \mu \mathrm{m})\end{array}$ & 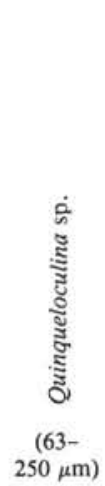 & 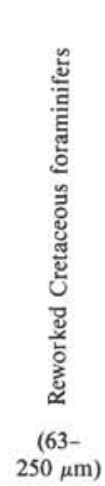 \\
\hline $13-1,30-34$ & $\mathrm{P}$ & $\mathbf{P}$ & $\mathrm{P}$ & $P$ & 69.8 & 12.8 & 5.8 & 50.0 & 7 & & \\
\hline $13-2,30-34$ & $\mathrm{P}$ & $\mathrm{P}$ & $\mathrm{P}$ & $\mathrm{P}$ & 57.6 & 58.6 & 76.5 & 25.3 & $6(4)$ & $\mathrm{R}$ & \\
\hline $13-3,30-34$ & $\mathrm{P}$ & $\mathrm{P}$ & $\mathrm{P}$ & $\mathrm{P}$ & 48.4 & 79.9 & 80.6 & 15.2 & 3 & $\mathrm{R}$ & \\
\hline $13-4,30-34$ & $\mathrm{P}$ & $\mathrm{P}$ & $\mathrm{P}$ & $\mathrm{P}$ & 51.4 & 58.6 & 76.5 & 31.0 & $6(4)$ & & \\
\hline $13-5,30-34$ & $\mathbf{P}$ & $\mathrm{P}$ & $\mathrm{P}$ & $\mathrm{P}$ & 36.0 & 95.1 & 92.3 & 17.8 & 3 & & \\
\hline $13-6,30-34$ & \multicolumn{11}{|c|}{ Too few specimens } \\
\hline $14-1,30-34$ & $\mathbf{P}$ & $\mathbf{P}$ & $\mathrm{P}$ & $\mathbf{P}$ & $54.3^{2}$ & 70.9 & 85.3 & 29.6 & $6(4)$ & $\mathbf{R}$ & \\
\hline $14-2,30-34$ & $\mathrm{P}$ & $\mathrm{P}$ & $\mathrm{P}$ & $\mathrm{P}$ & 52.6 & 87.0 & 89.6 & 43.7 & 6 & $\mathrm{R}$ & \\
\hline $14-3,30-34$ & $\mathrm{P}$ & $\mathrm{P}$ & $\mathrm{P}$ & $\mathrm{P}$ & 85.0 & 92.6 & 75.9 & 40.2 & 6 & & \\
\hline $14-4,30-34$ & $P$ & $\mathrm{P}$ & $\mathrm{P}$ & $\mathrm{P}$ & 68.9 & 89.9 & 83.3 & 41.7 & 6 & $\mathbf{R}$ & \\
\hline $15-1,30-34$ & $\mathrm{P}$ & $\mathrm{P}$ & $\mathrm{P}$ & $\mathrm{P}$ & 43.2 & 76.0 & 79.5 & 17.9 & 3 & $\mathbf{R}$ & \\
\hline $15-2,30-34$ & $\mathrm{P}$ & $\mathrm{P}$ & $\mathrm{P}$ & $\mathrm{P}$ & 44.8 & 68.8 & 90.6 & 33.3 & 4 & $\mathrm{R}$ & \\
\hline $15-3,30-34$ & P & $\mathrm{P}$ & $\mathrm{P}$ & $\mathrm{P}$ & 68.5 & 83.6 & 70.6 & 49.7 & 6 & $\mathrm{R}$ & \\
\hline $15-4,30-34$ & $\mathrm{P}$ & $\mathrm{P}$ & $\mathrm{P}$ & $\mathrm{P}$ & 41.8 & 85.1 & 86.3 & 38.7 & 4 & $\mathrm{R}$ & \\
\hline $15-5,30-34$ & $\mathrm{P}$ & P & $\mathrm{P}$ & $\mathrm{P}$ & 69.1 & 77.9 & 72.8 & 30.4 & 6 & & \\
\hline $15-6,30-34$ & $\mathrm{P}$ & $\mathrm{P}$ & $\mathrm{P}$ & $\mathrm{P}$ & 49.8 & 76.2 & 82.8 & 26.0 & 4 & & \\
\hline $16-1,30-34$ & $\mathrm{P}$ & P & $\mathrm{P}$ & P & 77.7 & 91.4 & 76.3 & 82.7 & 6 & $\mathrm{R}$ & \\
\hline $16-2,30-34$ & P & $\mathrm{P}$ & $\mathrm{P}$ & P & 74.0 & 88.7 & 80.3 & 61.8 & 6 & & \\
\hline $16-3,30-34$ & $\mathrm{P}$ & P & $\mathrm{P}$ & $\mathrm{P}$ & 69.5 & 83.6 & 71.9 & 63.9 & 6 & $\mathrm{R}$ & \\
\hline $16-4,30-34$ & $\mathrm{P}$ & $\mathrm{P}$ & $\mathrm{P}$ & $\mathrm{P}$ & 59.6 & 44.7 & 61.5 & 61.1 & 6 & $\mathrm{R}$ & \\
\hline $17-1,30-34$ & $\mathrm{P}$ & $\mathrm{P}$ & $\mathrm{P}$ & $\mathrm{P}$ & 61.4 & 92.8 & 96.9 & 13.3 & $6(3)$ & & \\
\hline $17-2,30-34$ & & & & & & & & & 10 & & \\
\hline $17-3,30-34$ & $P$ & $\mathrm{P}$ & $P$ & $P$ & 56.3 & 58.3 & 66.7 & 38.1 & $6(4)$ & & \\
\hline $17-4,30-34$ & $\mathrm{P}$ & $\mathrm{P}$ & $\mathrm{P}$ & $\mathrm{P}$ & 52.0 & 90.8 & 93.4 & 39.1 & $6(4)$ & $\mathrm{R}$ & \\
\hline $17-5,30-34$ & $P$ & $P$ & $\mathrm{P}$ & $\mathrm{P}$ & 27.3 & 98.5 & 99.0 & 20.9 & 4 & & \\
\hline $17-6,30-34$ & $\mathrm{P}$ & $\mathrm{P}$ & $\mathrm{P}$ & $\mathrm{P}$ & 50.0 & 59.8 & 65.6 & 46.3 & 6 & $\mathrm{R}$ & \\
\hline $18-1,30-34$ & $\mathrm{P}$ & $\mathrm{P}$ & $x$ & $\mathrm{P}$ & 100.0 & 50.0 & & & 9 & & P \\
\hline $19-2,30-34$ & $\mathrm{P}$ & $\mathrm{P}$ & $\mathrm{P}$ & P & 39.0 & 91.2 & 96.5 & 32.3 & 4 & C & P \\
\hline $19-3,30-34$ & $\mathrm{P}$ & $\mathrm{P}$ & $\mathrm{P}$ & $\mathrm{P}$ & 34.8 & 86.7 & 89.0 & 26.7 & 4 & & \\
\hline $19-4,30-34$ & $\mathrm{P}$ & $\mathrm{P}$ & $\mathrm{P}$ & P & 45.2 & 74.2 & 88.2 & 19.4 & 3 & C & \\
\hline $19-5,30-34$ & $\mathrm{P}$ & P & $\mathrm{P}$ & P & 31.0 & 88.2 & 92.1 & 21.9 & 3 & C & $P$ \\
\hline $19-6,30-34$ & $\mathrm{P}$ & $\mathrm{P}$ & $\mathrm{P}$ & $\mathrm{P}$ & 37.1 & 82.9 & 93.2 & 7.5 & 3 & & P \\
\hline $20-1,30-34$ & & & & & & & & & 10 & & \\
\hline $20-2,30-34$ & & & & & & & & & 10 & & \\
\hline $20-3,30-34$ & $\mathrm{P}$ & $\mathrm{P}$ & $\mathbf{P}$ & $\mathrm{P}$ & 61.6 & 84.9 & 78.6 & 22.2 & $6(3)$ & $\mathrm{C}$ & \\
\hline $20-4,30-34$ & \multicolumn{11}{|c|}{ Too few specimens } \\
\hline $20-5,30-34$ & & & & & & & & & 10 & & \\
\hline $21-1,30-34$ & & & & & & & & & 10 & & \\
\hline $21-2,30-34$ & $\mathrm{P}$ & $\mathrm{P}$ & $\mathrm{P}$ & $\mathrm{P}$ & 76.5 & 47.1 & & & 7 & & \\
\hline $21-3,30-34$ & $\mathrm{x}$ & $\mathrm{P}$ & $\mathrm{P}$ & $\mathrm{P}$ & 94.7 & 68.4 & 0 & & 8 & & \\
\hline $22-1,30-34$ & \multirow{2}{*}{\multicolumn{11}{|c|}{ Foraminifers present, not determinable }} \\
\hline $22-2,30-34$ & & & & & & & & & & & \\
\hline $22-3,30-34$ & & & & & & & & & 10 & & \\
\hline $22-4,30-34$ & & & & & & & & & 10 & & \\
\hline $22-5,30-34$ & & & & & & & & & 10 & & \\
\hline $22-6,30-34$ & $\mathrm{P}$ & $\mathrm{P}$ & $\mathrm{P}$ & $\mathbf{P}$ & 42.9 & 71.4 & 75.0 & 33.3 & 4 & & \\
\hline $22-7,30-34$ & & & & & & & & & 10 & & \\
\hline $23-1,30-34$ & & & & & & & & & 10 & & \\
\hline $23-2,30-34$ & & $\mathrm{P}$ & & $\mathrm{P}$ & 7.6 & 100.0 & 100.0 & 0.7 & 2 & & \\
\hline $23-3,95-97$ & $\mathrm{P}$ & $\mathrm{P}$ & $\mathrm{x}$ & $\mathrm{P}$ & 35.7 & 85.7 & 100.0 & 0 & 2 & $\mathbf{R}$ & \\
\hline $23-4,30-34$ & \multicolumn{11}{|c|}{ Too few specimens } \\
\hline $24-1,30-34$ & & & & & & & & & 10 & & \\
\hline $24-2,30-34$ & $\mathrm{P}$ & $P$ & $P$ & P & 12.3 & 96.9 & 98.7 & 27.7 & 4 & & \\
\hline $24-3,30-34$ & & & & & & & & & 10 & & \\
\hline $24-4,30-34$ & $\mathrm{P}$ & $P$ & $\mathrm{P}$ & $P$ & 21.4 & 92.9 & 95.5 & 16.7 & 3 & & \\
\hline $24-5,30-34$ & & & & & & & & & 10 & & \\
\hline $24-6,30-34$ & \multirow{2}{*}{\multicolumn{11}{|c|}{ Foraminifers present, not determinable }} \\
\hline $24-7,30-34$ & & & & & & & & & & & \\
\hline $25-1,30-32$ & & & & & & & & & 10 & & $\mathrm{P}$ \\
\hline $25-2,30-32$ & & P & & & & & & & 9 & & $\mathrm{P}$ \\
\hline
\end{tabular}


Table 2 (continued).

\begin{tabular}{|c|c|c|c|c|c|c|c|c|c|c|c|}
\hline $\begin{array}{c}\text { Sample } \\
\text { (Core-Section, } \\
\text { interval in } \mathrm{cm} \text { ) }\end{array}$ & 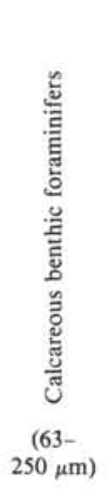 & 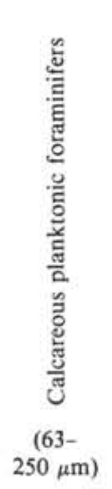 & 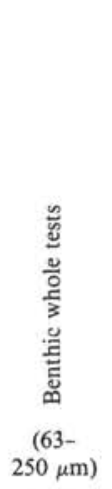 & 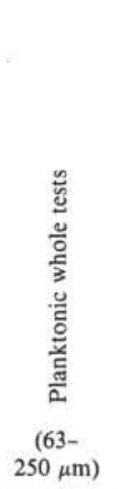 & 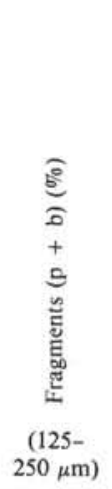 & 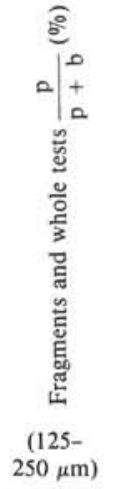 & 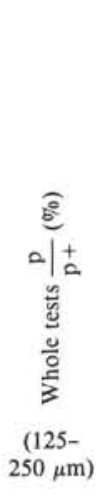 & $\begin{array}{c}\stackrel{Ð}{5} \\
\simeq \\
(125- \\
250 \mu \mathrm{m})\end{array}$ & $\begin{array}{c}\text { 号 } \\
(63- \\
250 \mu \mathrm{m})\end{array}$ & 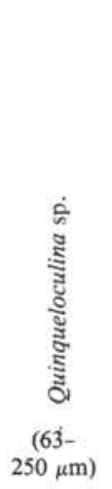 & 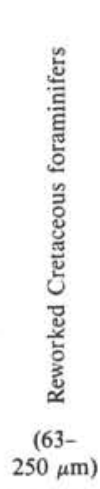 \\
\hline $25-3,30-32$ & & P & & & 100.0 & 100.0 & & 100.0 & 9 & & \\
\hline $25-4,30-32$ & P & P & P & P & 79.5 & 97.7 & 88.9 & 93.3 & 6 & & \\
\hline $25-5,30-32$ & P & P & P & P & 88.9 & 97.8 & 80.0 & 82.4 & 6 & & \\
\hline $25-6,30-32$ & $\mathrm{x}$ & P & & P & 100.0 & 100.0 & 100.0 & 100.0 & 9 & & $P$ \\
\hline $25-7,30-32$ & $x$ & P & & & 100.0 & 100.0 & 100.0 & 89.5 & 9 & & \\
\hline $26-1,30-34$ & & & & & & & & & 10 & & \\
\hline $26-2,22-26$ & & & & & & & & & 10 & & \\
\hline $26-3,30-34$ & & & & & & & & & 10 & & \\
\hline $26-4,32-36$ & & & & & & & & & 10 & & \\
\hline $26-5,32-36$ & & $x$ & & & & & & & 9 & & \\
\hline $26-6,32-36$ & P & P & $\mathrm{P}$ & & 77.8 & 55.6 & 0 & 100.0 & 8 & & \\
\hline $28-1,30-34$ & $\mathrm{x}$ & & & & 100.0 & 100.0 & 100.0 & 100.0 & 9 & & \\
\hline $29-1,30-34$ & & $\mathrm{x}$ & & & 100.0 & & & & 9 & & \\
\hline $29-2,30-34$ & & & & & & & & & 10 & & \\
\hline $29-3,30-34$ & P & P & & P & 83.8 & 97.3 & 100.0 & 68.8 & 6 & & \\
\hline $29-4,30-34$ & P & P & & & 100.0 & & & & 9 & & \\
\hline $29-5,30-34$ & & $\mathrm{P}$ & & & 100.0 & & & & 9 & & \\
\hline $30-1,60-64$ & & & & & & & & & 10 & & \\
\hline $30-2,60-64$ & & & & & & & & & 10 & & \\
\hline $30-3,60-64$ & P & P & & & 100.0 & & & & 9 & & \\
\hline $30-4,60-64$ & P & P & & & 100.0 & & & & 9 & & \\
\hline $31-1,37-39$ & & P & & & 100.0 & & & & 9 & & \\
\hline $31-2,37-39$ & & & & & & & & & 10 & & \\
\hline $31-3,37-39$ & & & & & & & & & 10 & & \\
\hline $32-1,55-57$ & & & & & & & & & 10 & & \\
\hline $32-2,94-96$ & P & P & P & P & 32.0 & 99.5 & 100.0 & 13.5 & 3 & & \\
\hline $33-1,30-34$ & & & & & & few specim & & & & & \\
\hline $33-2,30-34$ & & & & & & & & & 10 & & \\
\hline $33-3,30-34$ & & & & & & & & & 10 & & \\
\hline $33-4,30-34$ & P & $\mathrm{P}$ & & P & 54.5 & 72.7 & 100.0 & 21.4 & $6(3)$ & & \\
\hline $33-5,30-34$ & & P & & $\mathrm{x}$ & 100.0 & & & & 9 & & \\
\hline $33-6,30-34$ & P & P & P & P & 29.2 & 77.1 & 85.3 & 11.1 & 3 & & \\
\hline $34-1,35-38$ & & & & & & & & & 10 & & \\
\hline $34-2,35-38$ & P & P & $P$ & P & 44.3 & 71.3 & 82.4 & 25.9 & 4 & A & \\
\hline $34-3,35-38$ & P & $P$ & P & P & 71.8 & 60.9 & 75.5 & 81.1 & 6 & & \\
\hline $34-4,35-38$ & $\mathrm{x}$ & P & & P & 54.8 & 100.0 & 100.0 & 71.4 & 6 & & \\
\hline $35-1,30-33$ & $\mathrm{P}$ & P & P & $\mathrm{P}$ & 18.2 & 81.8 & 77.8 & 57.1 & 5 & & \\
\hline $35-2,30-33$ & P & P & $\mathrm{P}$ & P & 52.6 & 63.2 & 44.2 & 62.5 & 6 & & \\
\hline $35-3,30-33$ & P & $\mathrm{P}$ & P & P & 49.3 & 73.1 & 94.1 & 25.0 & 4 & & \\
\hline $35-4,30-33$ & P & P & P & P & 77.5 & 80.0 & 77.8 & 81.3 & 6 & & \\
\hline $35-5,30-33$ & & & & & & & & & 10 & & \\
\hline $35-6,30-33$ & P & P & $\mathrm{P}$ & P & 59.7 & 67.2 & 77.1 & 21.7 & $6(3)$ & & \\
\hline $36-1,28-32$ & P & P & P & P & 63.5 & 46.2 & 57.9 & 17.4 & $6(3)$ & & \\
\hline $36-2,30-34$ & P & $\mathrm{P}$ & $\mathrm{P}$ & $\mathrm{P}$ & 17.7 & 95.8 & 97.5 & 10.7 & 3 & & \\
\hline $36-3,28-32$ & P & P & P & P & 81.3 & 24.1 & 45.7 & 22.7 & $6(3)$ & A & \\
\hline $36-4,28-32$ & P & P & $\mathrm{P}$ & P & 42.3 & 88.5 & 96.7 & 3.2 & 2 & & \\
\hline $37-1,30-34$ & & $\mathrm{P}$ & & P & 36.4 & 100.0 & 100.0 & & (3) & & \\
\hline $37-2,30-34$ & P & $\mathrm{P}$ & P & $\mathrm{P}$ & 30.4 & 89.9 & 99.1 & 17.6 & 3 & & \\
\hline $38-1,30-34$ & $\mathrm{x}$ & $x$ & & & & & & & 10 & & \\
\hline $38-2,30-34$ & & & & & & & & & 10 & & \\
\hline $39-1,30-34$ & & & & & & & & & 10 & & \\
\hline $39-2,30-34$ & & & & & & & & & 10 & & \\
\hline $40-1,30-34$ & & & & & & & & & 10 & & \\
\hline $40-2,30-34$ & & & & & & & & & 10 & & \\
\hline $40-3,30-34$ & & & & & & & & & 10 & & \\
\hline $40-4,30-34$ & & & & & & & & & 10 & & \\
\hline $40-5,30-34$ & & & & & & & & & 10 & & \\
\hline $40-6,30-34$ & & & & & & & & & 10 & & \\
\hline $41-1,30-34$ & & & & & & & & & 10 & & \\
\hline $41-2,30-34$ & & & & & & & & & 10 & & \\
\hline
\end{tabular}




\begin{tabular}{|c|c|c|c|c|c|c|c|c|c|c|c|}
\hline $\begin{array}{c}\text { Sample } \\
\text { (Core-Section, } \\
\text { interval in } \mathrm{cm} \text { ) }\end{array}$ & 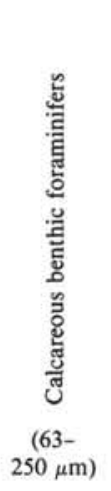 & 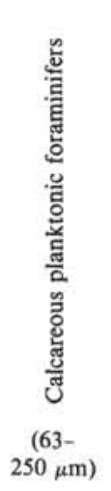 & 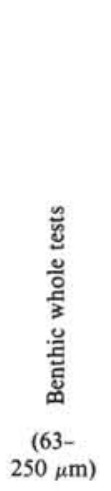 & 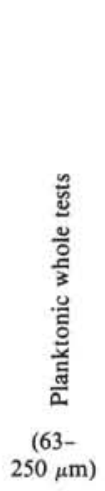 & 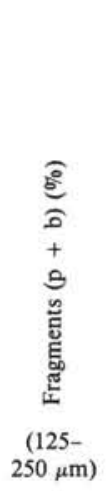 & 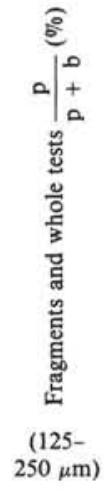 & 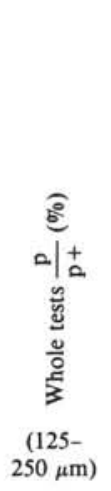 & 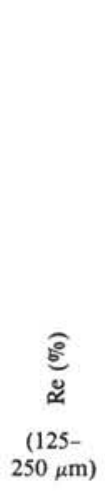 & $\begin{array}{c}\text { L } \\
(63- \\
250 \mu \mathrm{m})\end{array}$ & 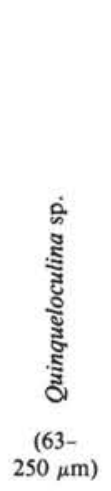 & 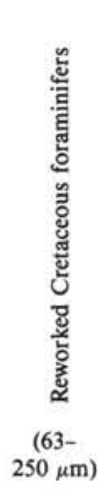 \\
\hline $41-3,30-34$ & & P & & $\mathbf{P}$ & & & & & 9 & & \\
\hline $41-4,30-34$ & & & & & & & & & 10 & & \\
\hline $41-5,30-34$ & & & & & & & & & 10 & & \\
\hline $41-6,30-34$ & P & $P$ & P & P & 83.0 & 94.7 & 91.2 & 60.6 & 6 & R & \\
\hline $42-1,30-34$ & & P & & P & 50.0 & 100.0 & 100.0 & 42.1 & 6 & & \\
\hline $42-2,30-34$ & & & & & & & & & 10 & & \\
\hline $42-3,30-34$ & & & & & & & & & 10 & & \\
\hline $42-4,30-34$ & & & & & & & & & 10 & & \\
\hline $42-5,30-34$ & P & $\mathrm{x}$ & P & P & 73.3 & 63.3 & 75.0 & 28.6 & $6(4)$ & & \\
\hline $43-1,30-34$ & P & P & & P & 66.7 & 93.3 & 100.0 & 58.3 & 6 & & \\
\hline $44-1,30-34$ & & & & & & & & & 10 & & \\
\hline $44-2,30-34$ & P & P & & & & & & & 9 & $\mathrm{R}$ & \\
\hline $44-3,30-34$ & & & & & & & & & 10 & & \\
\hline $44-4,30-34$ & & & & & & & & & 10 & & \\
\hline $45-1,30-34$ & & P & & & 100.0 & 100.0 & 100.0 & 100.0 & 9 & & \\
\hline $45-2,30-34$ & & $\mathrm{P}$ & & & 100.0 & & & & 9 & & \\
\hline $45-3,30-34$ & P & P & & & 100.0 & 94.4 & & 58.8 & 9 & & \\
\hline $45-4,30-34$ & & & & & & & & & 10 & & \\
\hline $45-5,30-34$ & & & & & & & & & 10 & & \\
\hline $46-1,30-34$ & & & & & & & & & 10 & & \\
\hline $46-2,30-34$ & & & & & & & & & 10 & & \\
\hline $46-3,30-34$ & & & & & & & & & 10 & & \\
\hline $46-4,30-34$ & & & & & & & & & 10 & & \\
\hline $48-1,42-44$ & & & & & & & & & 10 & & \\
\hline $49-1,30-34$ & & & & & & & & & 10 & & \\
\hline $49-2,30-34$ & & & & & & & & & 10 & & \\
\hline $49-3,30-34$ & & & & & & & & & 10 & & \\
\hline $50-1,30-34$ & & & & & & & & & 10 & & \\
\hline $50-2,30-34$ & & & & & & & & & 10 & & \\
\hline $50-3,30-34$ & & & & & & & & & 10 & & \\
\hline $50-4,30-34$ & & & & & & & & & 10 & & \\
\hline $51-1,30-34$ & & & & & & & & & 10 & & \\
\hline $51-2,30-34$ & & & & & & & & & 10 & & \\
\hline $52-1,30-34$ & & & & & & & & & 10 & & \\
\hline $52-2,30-34$ & & & & & & & & & 10 & & \\
\hline $52-3,30-34$ & & & & & & & & & 10 & & \\
\hline $52-4,30-34$ & & & & & & & & & 10 & & \\
\hline $52-5,30-34$ & & & & & & & & & 10 & & \\
\hline $53-1,30-34$ & & & & & & & & & 10 & & \\
\hline $53-2,30-34$ & & & & & & & & & 10 & & \\
\hline $54-1,30-34$ & P & P & & & 100.0 & & & & 9 & & \\
\hline
\end{tabular}

Note: $\mathrm{p}=$ planktonic foraminifers; $\mathrm{b}=$ benthic foraminifers; $\mathrm{Re}=$ resistant planktonic foraminifers; $\mathrm{FS}=$ foraminiferal dissolution code. $\mathrm{P}=$ present; $\mathrm{R}=$ rare, $0.1-<3.0 ; \mathrm{C}=$ common, $3.0-<10.0 ; \mathrm{A}=$ abundant, $10.0-40.0 ; \mathrm{D}=$ dominant, $40.0-100.0 ; \mathrm{X}:$ only in fraction $<125 \mu \mathrm{m}$. The foraminiferal percentage was calculated for the fractions between 125 and $250 \mu \mathrm{m}$. The last two columns show the samples with displaced Quinqueloculina sp., a shallow-water benthic foraminifer, and samples with reworked Cretaceous foraminifers.

North Atlantic this boundary does not coincide with the first appearance of Globorotalia margaritae (Berggren et al., 1985), so other indicators or events must be used to fix this boundary. The influx of cold-water fauna in Sample 603-16-1, 30-34 cm (Globigerina bulloides d'Orbigny, Neogloboquadrina pachyderma [Ehrenberg], and Neogloboquadrina atlantica [Berggren]) is such an event. As noted previously, it is preceded by the entrance of Globorotalia praemiocenica Lamb and Beard in Sample 603-16-2, 30-34 cm and Globigerinoides conglobatus (Brady) in Sample 603-17-4, 30-34 cm.
The first occurrence of $G$. conglobatus in the section precedes a sudden short rise in CCD level in Sample 603-17-2, 30-34 cm. Hemleben and Auras (1984) found the same pattern at Site 541 of Leg 78A in the Central Atlantic, where the first occurrence of $G$. conglobatus was found below the Miocene/Pliocene boundary, based on nannofossils (Bergen, 1984).

Under the assumption that the cold-water sediment of Sample $603-16-1,30-34 \mathrm{~cm}$ is deposited during the generally accepted Messinian cooling period, the Miocene/Pliocene boundary of Site 603 may be placed above 
Core 16. This is in accordance with findings by Moullade in Site 604 (this volume), where a sequence of events similar to those at Site 603 occurs: the first appearance of $G$. conglobatus, followed by the first appearance of Globorotalia praemiocenica and the last appearance of a cold-water planktonic species (Globigerina praebulloides). Moullade draws the Miocene/Pliocene boundary at Site 604 just above the last occurrence of $G$. praebulloides (Muza et al., this volume); at Site 603 the Miocene/Pliocene boundary can also be drawn between Cores 16 and 15, on the basis of nannofossil evidence (Muza et al., this volume).

\section{CONCLUSIONS}

The beginning of the fluctuations in the dissolution curve coincides with seismic reflection Horizon X, which marks the onset of the present WBUC in the middle Miocene.

Major seismic sequence boundaries and important lithological unit boundaries correlate with fluctuations in the dissolution curve caused by variations of the WBUC contour current and/or fluctuations in CCD level.

It has taken Site 603 approximately $6.6 \mathrm{~m}$.y. to rise permanently above the CCD, its sedimentary record potentially being a high-resolution monitor of CCD fluctuations. Unfortunately, faunal displacement and reworking disturb the signal.

The Miocene/Pliocene boundary may be placed above Core 16 , on the basis of paleontologic and paleoceanographic evidence.

\section{ACKNOWLEDGMENTS}

I thank Prof. Dr. J. E. van Hinte from the Vrije Universiteit for the initial idea for this chapter, the material for this study, and for review- ing the manuscript. I also thank the Leg 93 scientific and technical party for sampling and Dr. W. H. Drucker from the University of Amsterdam for the X-ray analysis.

\section{REFERENCES}

Bergen, J. A., 1984. Calcareous nannoplankton from Deep Sea Drilling Project Leg 78A: evidence for imbricate underthrusting at the Lesser Antillian Active Margin. In Biju-Duval, B., Moore J. C., et al., Init. Repts. DSDP, 78A: Washington (U.S. Govt. Printing Office), 411-445.

Berger, W. H., 1979. Preservation of Foraminifera: Houston (Soc. Econ. Paleontol. Mineral.), Short Course No. 6:105-155.

Berger, W. H., and von Rad, U., 1972. Cretaceous and Cenozoic sediments from the Atlantic Ocean. In Hayes, D. E., Pimm, A. C., et al., Init. Repts. DSDP, 14: Washington (U.S. Govt. Printing Office), 787-954.

Berggren, W. A., Kent, D. V., and Van Couvering, J. A., 1985. The Neogene: Part 2. Neogene geochronology and chronostratigraphy. In Snelling, N. J. (Ed.), The Geochronology of the Geological Record: London (Blackwell Sci. Publ.), Geol. Soc. Mem., 10:211-250.

Blow, W. H., 1969. Late middle Eocene to Recent planktonic foraminiferal biostratigraphy. Proc. 1st. Int. Conf. Planktonic Microfossils, Geneva (1967), 1:199-442.

Hemleben, C., and Auras, A., 1984. Variations in the calcite dissolution on the Barbados Ridge complex at Sites 541 and 543, Deep Sea Drilling Project Leg 78A. In Biju-Duval, B., Moore J. C., et al., Init. Repts. DSDP, 78A: Washington (U.S. Govt. Printing Office), 471-497.

Stainforth, R. M., Lamb, J. L., Beard, J. H., and Jeffords, R. M., 1975. Cenozoic planktonic foraminiferal zonation and characteristics of index forms. Univ. of Kansas, Paleontol. Contrib., Art. 62.

Tucholke B. E., and Vogt P. R., 1979. Western North Atlantic: sedimentary evolution and aspects of tectonic history. In Tucholke, B. E., Vogt, P. R., et al., Init. Repts. DSDP, 43: Washington (U.S. Govt. Printing Office), 791-825.

Date of Initial Receipt: 9 April 1985

Date of Acceptance: 13 May 1986 
Table 3. Estimated percentages of washed residue components of samples from Cores 54 to 13 of Hole 603 .

\begin{tabular}{|c|c|c|c|c|c|c|c|c|c|c|c|c|c|c|c|c|}
\hline \multirow{4}{*}{$\begin{array}{c}\text { Sample } \\
\text { (Cote-Seetion, } \\
\text { interval in } \mathrm{cm} \text { ) }\end{array}$} & \multicolumn{12}{|c|}{ Minerals } & \multirow{2}{*}{\multicolumn{4}{|c|}{$\begin{array}{c}\text { Fossils } \\
\text { Calcareous }\end{array}$}} \\
\hline & \multirow{2}{*}{\multicolumn{2}{|c|}{$\begin{array}{c}\text { Sedimentary rock } \\
\text { fragments (including } \\
\text { mica, clay, sitit) } \\
\end{array}$}} & \multirow{2}{*}{\multicolumn{2}{|c|}{$\frac{\mathrm{g}}{\mathrm{g}}$}} & \multirow{2}{*}{\multicolumn{2}{|c|}{ 善量 }} & \multirow{2}{*}{\multicolumn{2}{|c|}{ 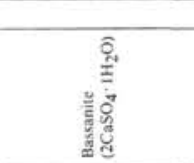 }} & \multirow{2}{*}{\multicolumn{2}{|c|}{ 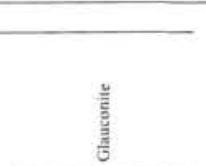 }} & \multirow{2}{*}{\multicolumn{2}{|c|}{ 형 }} & & & & \\
\hline & & & & & & & & & & & & & \multicolumn{2}{|c|}{$\frac{5}{\frac{5}{2}}$} & 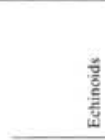 & \\
\hline & $\begin{array}{c}(125- \\
<250 \mathrm{~mm})\end{array}$ & 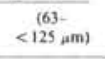 & $\begin{array}{l}\quad(125) \\
<250 \mathrm{\mu m})\end{array}$ & $\frac{163-}{<125 \mathrm{am})}$ & 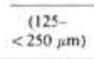 & $\begin{array}{l}<163- \\
<125 \mu \mathrm{m})\end{array}$ & $\begin{array}{c}125- \\
<250 \mathrm{~km})\end{array}$ & $\frac{(63-}{<125 \mathrm{\mu m})}$ & $\begin{array}{l}125- \\
<250 \mu \mathrm{m})\end{array}$ & $\begin{array}{c}<125 \mathrm{sm}- \\
<125 \mathrm{~m})\end{array}$ & $\begin{array}{l}(125) \\
<250 \mu \mathrm{m})\end{array}$ & $\frac{(63-}{<125 \mathrm{sm})}$ & $\begin{array}{c}125- \\
<250 \mu \mathrm{m})\end{array}$ & $\begin{array}{l}(63- \\
<125 \mathrm{~mm})\end{array}$ & $\begin{array}{c}125- \\
<250 \mathrm{pm})\end{array}$ & $\begin{array}{c}(63-) \\
\langle 125 \mathrm{~mm})\end{array}$ \\
\hline $13-1,30-34$ & $\begin{array}{l}15.0 \\
150\end{array}$ & 37.5 & 4.0 & 4.0 & 56.0 & 35.0 & 16.0 & & & & & 0.5 & 8.7 & 19.0 & & \\
\hline $\begin{array}{l}13 \cdot 2,30-34 \\
13-3,30-34\end{array}$ & $\begin{array}{r}15.0 \\
7.0\end{array}$ & $\begin{array}{l}20,0 \\
21.5\end{array}$ & $\begin{array}{l}4.5 \\
2.8\end{array}$ & $\begin{array}{l}7.0 \\
4.0\end{array}$ & $\begin{array}{l}60.0 \\
60.0\end{array}$ & $\begin{array}{l}60.0 \\
55.0\end{array}$ & 19.0 & & & & & 0.1 & $\begin{array}{l}1.4 \\
30.0\end{array}$ & $\begin{array}{l}12.0 \\
18.0\end{array}$ & . & - \\
\hline 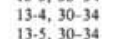 & & 0.8 & 20 & . & 98.5 & $\begin{array}{l}95.0 \\
880\end{array}$ & & & & & & - & 1.5 & 4.0 & & \\
\hline $\begin{array}{l}13.5 .53-3-34 \\
13 \cdot 6,30-34\end{array}$ & 65.0 & $\begin{array}{l}1.0 \\
61.5\end{array}$ & $\begin{array}{l}7.0 \\
20.0\end{array}$ & $\begin{array}{l}10.5 \\
27.5\end{array}$ & $\begin{array}{r}62.0 \\
7.0\end{array}$ & $\begin{array}{l}85.0 \\
10.0\end{array}$ & s.o & & & & & $\begin{array}{l}0.5 \\
0.4\end{array}$ & ${ }^{1.0}$ & 1.5 & & \\
\hline $\begin{array}{l}14 \cdot 1,30-34 \\
4 \cdot 2,30-34\end{array}$ & 20 & $\begin{array}{l}1.5 \\
3.0\end{array}$ & $\begin{array}{l}7.0 \\
0.4\end{array}$ & 22.0 & $\begin{array}{l}45.0 \\
780\end{array}$ & $\begin{array}{l}55.0 \\
490\end{array}$ & 28.0 & & & • & $\cdot$ & 0.1 & $\begin{array}{l}20.0 \\
15.0\end{array}$ & $\begin{array}{l}20.0 \\
430.0\end{array}$ & : & 0.1 \\
\hline 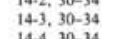 & & $\begin{array}{l}3.0 \\
1,0\end{array}$ & & & $\begin{array}{l}78.0 \\
45.0\end{array}$ & $\begin{array}{l}490.0 \\
40.0\end{array}$ & & & & : & & 0.1 & $\begin{array}{l}73.0 \\
49.0\end{array}$ & $\begin{array}{l}53.0 \\
58.0\end{array}$ & & \\
\hline $14 \cdot 4,30-34$ & 1.0 & 2.0 & 0.5 & 1.0 & 55.0 & 45.0 & 1.5 & & & & & . & 37.0 & 50.0 & & \\
\hline $15-1,30-34$ & & 7.0 & & & 50.0 & 70.0 & & & & 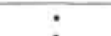 & . & 12 & 1.5 & 18.0 & & \\
\hline $\begin{array}{l}15-2,30-34 \\
15-3,30-34\end{array}$ & 2.5 & $\begin{array}{r}21.5 \\
3.5\end{array}$ & 5.0 & 13.0 & 50.0 & 30.0 & 25.0 & & & - & & 1.5 & 7.2 & 10.0 & & \\
\hline $15-4,30-34$ & & $\begin{array}{l}3.0 \\
1.5\end{array}$ & 3.4 & $\begin{array}{l}0.3 \\
1.5\end{array}$ & $\begin{array}{l}55.0 \\
75.0\end{array}$ & $\begin{array}{l}50.0 \\
50.0\end{array}$ & $\begin{array}{l}2.0 \\
3.5\end{array}$ & & & & & $\cdot$ & $\begin{array}{l}40.0 \\
18.0\end{array}$ & $\begin{array}{l}45.0 \\
460\end{array}$ & & \\
\hline $15-5,30-34$ & & & 7.0 & 23.5 & 50.0 & 60.0 & 30.0 & & & & & - & 12.9 & 14.0 & & \\
\hline $15-6,30-34$ & 1.5 & 1.0 & 6.0 & 20.0 & 65.0 & 65.0 & 18.0 & & & & & $\cdot$ & 9.5 & 13.0 & & \\
\hline $16-1,30-34$ & & 0.4 & & & 5.0 & 10.0 & & & & & - & : & 85.0 & 80.0 & . & $\cdot$ \\
\hline $\begin{array}{l}16-2,2,30-34 \\
16-3,30-34\end{array}$ & & $i, 2$ & & & $\begin{array}{l}{ }_{3}^{34.0} \\
70.0\end{array}$ & $\begin{array}{l}14.9 \\
60.0\end{array}$ & & & & & & $\dot{0.1}_{1}$ & $\begin{array}{l}65.0 \\
29.9\end{array}$ & $\begin{array}{l}85.0 \\
38.0\end{array}$ & . & . \\
\hline $\begin{array}{l}16-4,30-34 \\
1043\end{array}$ & & $\begin{array}{l}1.2 \\
1.0\end{array}$ & & & $\begin{array}{l}70.0 \\
94.0\end{array}$ & $\begin{array}{l}60.0 \\
78.0\end{array}$ & & & & & 0.1 & $\begin{array}{l}0.1 \\
1.0\end{array}$ & $\begin{array}{r}28.9 \\
5.0\end{array}$ & $\begin{array}{l}38.0 \\
18.0\end{array}$ & is & $\theta^{\circ}$ \\
\hline $77-1,30-34$ & & 1.4 & & & 93.5 & 56.0 & & & & & . & • & 6.0 & 42.0 & 0.2 & \\
\hline $17 \cdot 2,30-34$ & 40.0 & $\begin{array}{l}31.0 \\
40.0\end{array}$ & $\begin{array}{l}25,5 \\
150\end{array}$ & 15.0 & 30.0 & 54.0 & & & & & & $\therefore$ & 10 & 36 & & \\
\hline $\begin{array}{r}17-3,30-34 \\
17.430-34\end{array}$ & $\begin{aligned} 24.0 \\
0.5\end{aligned}$ & $\begin{array}{r}{ }^{40.0} \\
3.0\end{array}$ & $\begin{array}{l}15.0 \\
12\end{array}$ & $\begin{array}{l}5.0 \\
10\end{array}$ & 40.0 & $\begin{array}{l}50.0 \\
\$ \$ 0\end{array}$ & 10.0 & & & & & 0.5 & $\begin{array}{l}1.0 \\
280\end{array}$ & $\begin{array}{r}3.6 \\
400\end{array}$ & & . \\
\hline $17-5,30-34$ & & $\begin{array}{l}3.0 \\
\text { t. }\end{array}$ & & & $\begin{array}{l}60.0 \\
70.0\end{array}$ & $\begin{array}{l}55.0 \\
75.0\end{array}$ & & & & . & & is & 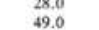 & $\begin{array}{l}{ }_{20.0} 21.5 \\
2.0\end{array}$ & & ${ }^{\circ}$ \\
\hline $17-6,30-34$ & 25.0 & 25.0 & 12.0 & 9.0 & 40.0 & 48.5 & 3.0 & & & & & 0.4 & 15.0 & 15.0 & & \\
\hline $18-1,30-34$ & 0.5 & 0.9 & & 1.5 & 99.2 & 97.0 & & & & & & 0.1 & 0.2 & 0.4 & & \\
\hline $19-2,30-34$ & 5.0 & 2.9 & 4.0 & 6.0 & 81.0 & 84.0 & 6.0 & & & & & 3.0 & 4.0 & 5.0 & & \\
\hline $19-3,30-34$ & 5.2 & 2.2 & 0.2 & 2.0 & 80.0 & 80.0 & & & & & & 0.5 & 4.0 & 15.0 & & 0.1 \\
\hline $\begin{array}{l}199.4,30-34 \\
19-5,30-34\end{array}$ & 200 & $\begin{array}{r}2.0 \\
160\end{array}$ & & & $\begin{array}{l}75.0 \\
600\end{array}$ & $\begin{array}{l}60.0 \\
50.0\end{array}$ & & & & & & 1.0 & $\begin{array}{l}24.0 \\
130\end{array}$ & $\begin{array}{l}37.0 \\
300\end{array}$ & & \\
\hline $19-6,30-34$ & 0.1 & 1.0 & 0.8 & & 800 & 730.0 & & & & & & 0.5 & 19.0 & 25.0 & 0.1 & \\
\hline $20-1,30-34$ & 3.0 & 40.5 & 0.5 & 2.0 & 96.0 & 55.0 & 0.4 & & & & & 2,0 & & & & \\
\hline $\begin{array}{l}20-2,2,30-34 \\
20-3,30-34\end{array}$ & $\begin{array}{r}1,3 \\
22.4\end{array}$ & $\begin{array}{l}13.0 \\
26.0\end{array}$ & & & $\begin{array}{l}98.8 \\
55.0\end{array}$ & $\begin{array}{l}\begin{array}{l}70.0 \\
50.0\end{array} \\
5\end{array}$ & & & & & 0.1 & $\begin{array}{l}8.0 \\
2.0\end{array}$ & 22.0 & 14.1 & & \\
\hline $\begin{array}{l}20-4,30-34 \\
20-5,30-34\end{array}$ & $\begin{array}{r}20.0 \\
100.0\end{array}$ & $\begin{array}{r}19.0 \\
100.0\end{array}$ & & & 80.0 & 80.0 & & & & & & 0.4 & 0.2 & & & \\
\hline $21-1,30-34$ & 40.0 & 40.0 & 4.0 & 0.9 & 50.0 & 50.0 & 6.0 & & & & & & & & & \\
\hline $\begin{array}{l}21 \cdot 2,30-34 \\
21 \cdot 3,30-34\end{array}$ & $\begin{array}{l}19.6 \\
6.5\end{array}$ & $\begin{array}{l}22.0 \\
15.9\end{array}$ & 2.0 & & $\begin{array}{l}80.0 \\
90.0\end{array}$ & $\begin{array}{l}75.0 \\
80.0\end{array}$ & & & & & 0.1 & $\begin{array}{l}0.8 \\
0.1\end{array}$ & $\begin{array}{l}0.1 \\
1.0\end{array}$ & 1.0 & & \\
\hline $22-1,30-34$ & 19.0 & 18.0 & 0.3 & 1.0 & 80.0 & 78.0 & 0.2 & & & & & 1.5 & & & & \\
\hline $\begin{array}{l}22-2,30-34 \\
22,3\end{array}$ & 1.0 & $\begin{array}{l}20.0 \\
45 .\end{array}$ & 02 & 1.0 & 95,0 & 66.0 & & & & & 0.1 & 1.0 & 3.5 & 8.0 & & \\
\hline $\begin{array}{l}22-3,3,3-3-34 \\
22-4,30-34\end{array}$ & $\begin{array}{l}50.0 \\
24.0\end{array}$ & $\begin{array}{l}45.5 \\
21.0\end{array}$ & $\begin{array}{l}0.2 \\
0.9\end{array}$ & 0.5 & $\begin{array}{l}45,5 \\
75,0\end{array}$ & $\begin{array}{l}50.0 \\
72.5\end{array}$ & & & & & & $\begin{array}{l}2.3 \\
3.0\end{array}$ & & & & \\
\hline $\begin{array}{l}22 \cdot 5,30-34 \\
22.60\end{array}$ & 0.9 & 9.0 & 0.5 & 9.0 & 90.0 & 77.0 & 0.4 & & & & 0.1 & 0.5 & 7 & 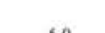 & & \\
\hline $\begin{array}{l}22-2,6,30-34 \\
22-7,30-34\end{array}$ & $\begin{array}{l}22.3 \\
50.0\end{array}$ & $\begin{array}{l}39.0 \\
48.0\end{array}$ & 1.5 & 7.0 & $\begin{array}{l}70.0 \\
48.0\end{array}$ & $\begin{array}{l}50.0 \\
43.0\end{array}$ & 0.4 & & & & & 1.0 & 7.0 & 6.0 & & \\
\hline $23 \cdot 1,30-34$ & 0.3 & 2.9 & 30.0 & 30.0 & 55.0 & 65.0 & 4.5 & 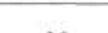 & & & & 0.1 & & & & \\
\hline $\begin{array}{l}23-2,3,30-34 \\
32-3,95-97\end{array}$ & & $\begin{array}{l}0.9 \\
0.7\end{array}$ & $\begin{array}{l}2.0 \\
0.4\end{array}$ & $\begin{array}{l}5.0 \\
6.0\end{array}$ & $\begin{array}{l}94.0 \\
950\end{array}$ & $\begin{array}{l}80.0 \\
91.0\end{array}$ & 2.8 & 3.0 & & & & 0.1 & ${ }_{03}^{1.0}$ & 6.0 & & \\
\hline $\begin{array}{l}23-3,3,93-97 \\
23-4,30-34\end{array}$ & $\begin{array}{l}3.6 \\
0.9\end{array}$ & $\begin{array}{l}0.7 \\
9.4\end{array}$ & $\begin{array}{l}0.4 \\
15.0\end{array}$ & $\begin{array}{r}60.0 \\
20.0\end{array}$ & $\begin{array}{l}95.0 \\
74.0\end{array}$ & $\begin{array}{l}\begin{array}{l}91.0 \\
70.0\end{array} \\
7\end{array}$ & $\begin{array}{l}0.1 \\
10.0\end{array}$ & & & & & & & $\begin{array}{l}0.3 \\
0.1\end{array}$ & & \\
\hline $24,1,30-34$ & & 5.0 & 5.0 & 6.0 & 91.0 & 86.0 & 4.0 & & & & & & 01 & 25s & & \\
\hline $\begin{array}{l}24,2,30-34 \\
24,3,30-34\end{array}$ & $\begin{array}{r}0.1 \\
46.0\end{array}$ & $\begin{array}{l}10.0 \\
45.4\end{array}$ & $\begin{array}{r}36.2 \\
20\end{array}$ & $\begin{array}{l}11.0 \\
9.0\end{array}$ & $\begin{array}{l}52,0 \\
460\end{array}$ & $\begin{array}{l}40.0 \\
450\end{array}$ & $\begin{array}{l}10.0 \\
\zeta 9\end{array}$ & & & & & 0.2 & 0.1 & 35.0 & & \\
\hline $24-4,30-34$ & 1.1 & 1.0 & 10.0 & 12.5 & $\begin{array}{l}80.0 \\
80.0\end{array}$ & 85.0 & 8.0 & & & & & & 0.2 & 0.5 & 0.1 & \\
\hline $245,30-34$ & 10.0 & 6.0 & 9.0 & 15.0 & 79.0 & 78.0 & 1.0 & & & & & & & & & \\
\hline $\begin{array}{l}24-6,30-34 \\
24,7,30-34\end{array}$ & $\begin{array}{l}1.8 \\
70.8\end{array}$ & $\begin{array}{r}22.5 \\
s .0\end{array}$ & $\begin{array}{r}45.0 \\
1.0\end{array}$ & $\begin{aligned} 35.0 \\
15.5\end{aligned}$ & $\begin{array}{l}{ }_{28.0}^{48.0} \\
28.0\end{array}$ & 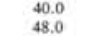 & 4.0 & & & & & $\dot{0.1}$ & $\begin{array}{l}0.1 \\
0.1\end{array}$ & $\begin{array}{l}0.1 \\
0.1\end{array}$ & & \\
\hline $25-1.30-32$ & $\begin{array}{l}30.0 \\
300\end{array}$ & 60.0 & 4 & 250 & 30.0 & 39.5 & & & & & & 0.1 & & & & \\
\hline $\begin{array}{l}25-2,30-32 \\
25-3,30-32\end{array}$ & $\begin{array}{l}30.0 \\
47.0\end{array}$ & $\begin{array}{l}\begin{array}{l}25.0 \\
43.0\end{array} \\
4\end{array}$ & 4.7 & 25.0 & $\begin{array}{l}{ }_{55.0}^{55.0} \\
50 .\end{array}$ & $\begin{array}{l}47.5 \\
50.0\end{array}$ & & & & & & $\begin{array}{l}0.1 \\
0.4\end{array}$ & $\begin{array}{l}0.1 \\
0.9\end{array}$ & $\begin{array}{l}0.1 \\
2.0\end{array}$ & & \\
\hline & 80.0 & 60.0 & & & 18.0 & 35.0 & & & & & & 0.1 & 0.9 & 2.0 & & \\
\hline $25-5,30-32$ & 53.0 & 50.0 & 0.1 & 0.4 & 45.0 & 47.0 & 0.1 & & & & 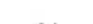 & 0.1 & 0.7 & 1.0 & & \\
\hline $\begin{array}{l}25-6,30-32 \\
25-7,30-32\end{array}$ & $\begin{array}{c}50.0 \\
0.9\end{array}$ & $\begin{array}{l}45.6 \\
2.1\end{array}$ & $\begin{array}{l}0.1 \\
0.5\end{array}$ & $\begin{array}{l}1.0 \\
0.2\end{array}$ & $\begin{array}{l}49.0 \\
99.5\end{array}$ & $\begin{array}{l}50.0 \\
90.0\end{array}$ & & & & & 0.1 & $\begin{array}{l}1.0 \\
0.1\end{array}$ & $\begin{array}{l}0.5 \\
1.5\end{array}$ & $\begin{array}{l}1.0 \\
1.0\end{array}$ & & \\
\hline $26-1,30-34$ & 41.5 & 20.4 & & & 55.0 & 53.0 & & & & & & & & & & \\
\hline $26-2,22-26$ & 6.1 & 4.0 & 10.0 & & 75.0 & 70.0 & 7.5 & & & & & & & & & \\
\hline $\begin{array}{l}28-3-3,30-34 \\
26-4,32-36\end{array}$ & $\begin{array}{r}49.0 \\
4.1\end{array}$ & $\begin{array}{l}35.2 \\
10.3\end{array}$ & 0.3 & 15 & $\begin{array}{l}50.0 \\
950.0\end{array}$ & $\begin{array}{l}49.0 \\
86.0\end{array}$ & & & & & & & & & & \\
\hline $\begin{array}{l}26-5,5,32-36 \\
26-6,32-36\end{array}$ & $\begin{array}{r}3.0 \\
10.5\end{array}$ & $\begin{array}{l}40 \\
10.3\end{array}$ & 8.0 & $\begin{array}{r}12.0 \\
5.0\end{array}$ & $\begin{array}{l}75.0 \\
700\end{array}$ & $\begin{array}{l}67.0 \\
680\end{array}$ & 1.0 & & & & 0 & & 0.1 & 01 & & \\
\hline$\frac{28-6,32-36}{28-1,30-34}$ & $\frac{10.5}{23.6}$ & $\frac{10.3}{45.0}$ & 3.0 & & 70.0 & $\frac{58,0}{53.0}$ & & & & & & 0.1 & 0.1 & 0.1 & & \\
\hline & & 36.0 & & 0.1 & & & 0.1 & & & & & & 0.7 & & & \\
\hline $\begin{array}{l}29 \cdot 2,3,30-34 \\
29-3,30-34\end{array}$ & $\begin{array}{l}49.0 \\
30.0\end{array}$ & $\begin{array}{l}34.5 \\
15.0\end{array}$ & 0.3 & 2.0 & $\begin{array}{l}50.0 \\
60.0\end{array}$ & $\begin{array}{l}60.0 \\
65.0\end{array}$ & & & & & & & 4.5 & 5.0 & & \\
\hline $29.4,30-34$ & 15.0 & 15.0 & & & 75.0 & 65.0 & & & & & & & 0.1 & 1.0 & & \\
\hline $29.5,30-34$ & 0.2 & 2.0 & & & 90.0 & 70.0 & & & & & & 0.1 & 0.1 & 0.4 & & \\
\hline $\begin{array}{l}30-1,60-64 \\
30-2,00-64\end{array}$ & $\begin{array}{l}12.0 \\
19.5\end{array}$ & $\begin{array}{l}6.5 \\
3.0\end{array}$ & 12.0 & & $\begin{array}{l}75.0 \\
800\end{array}$ & $\begin{array}{l}77,0 \\
50.0\end{array}$ & 0.4 & & & & & 0.5 & & & & \\
\hline
\end{tabular}


MIOCENE DISSOLUTION FACIES, SITE 603

Table 3 (continued).

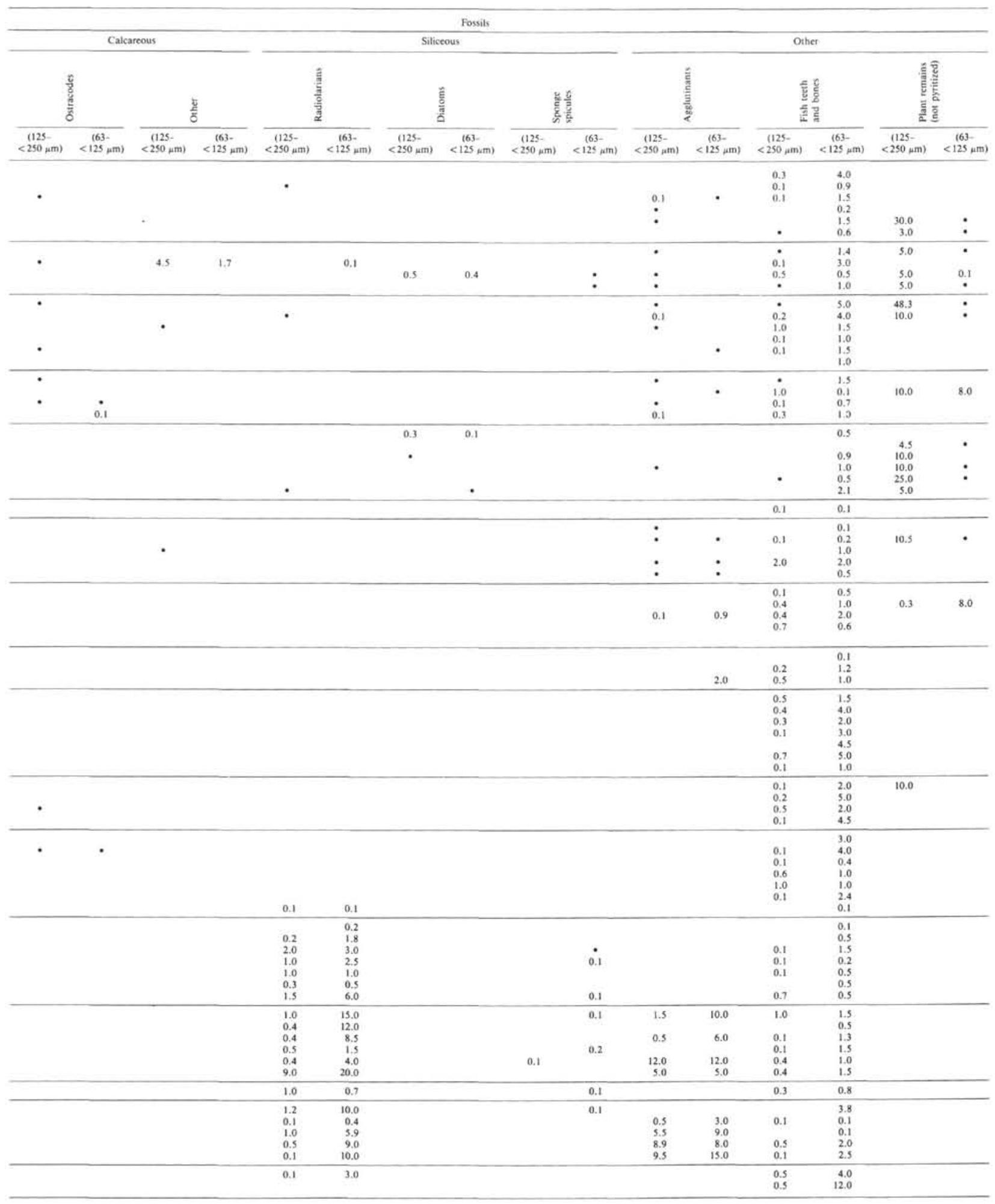

519 
Table 3 (continued).

\begin{tabular}{|c|c|c|c|c|c|c|c|c|c|c|c|c|c|c|c|c|}
\hline \multirow{4}{*}{$\begin{array}{l}\text { Sample } \\
\text { (Core-section, } \\
\text { interval in } \mathrm{cm} \text { ) }\end{array}$} & \multirow{3}{*}{\multicolumn{2}{|c|}{$\begin{array}{l}\text { Sedimentary rock } \\
\text { fragments (including } \\
\text { mica, clay, silt) }\end{array}$}} & & \multirow{2}{*}{\multicolumn{4}{|c|}{$\frac{\text { Fossils }}{\text { Calcareous }}$}} \\
\hline & & & \multicolumn{10}{|c|}{ Minerals } & & & & \\
\hline & & & \multicolumn{2}{|c|}{ 晷 } & \multicolumn{2}{|c|}{ 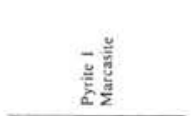 } & . & $\begin{array}{l}0 \\
0 \\
0\end{array}$ & & हैّ & \multicolumn{2}{|c|}{ 童 } & \multicolumn{2}{|c|}{ 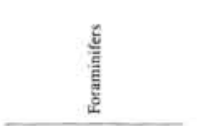 } & \multicolumn{2}{|l|}{ 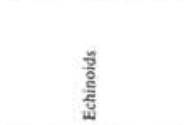 } \\
\hline & $\begin{array}{l}(125- \\
<250 \mu \mathrm{m})\end{array}$ & $\begin{array}{l}<125 \mu \mathrm{mm}) \\
<\frac{163 .}{2}\end{array}$ & $\begin{array}{l}(125- \\
<250 \mu \mathrm{mm})\end{array}$ & 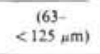 & $\begin{array}{l}(125- \\
<250 \mu \mathrm{m})\end{array}$ & $\begin{array}{l}(63-2) \\
<125 \mu \mathrm{m})\end{array}$ & $\begin{array}{l}<125- \\
<250 \mu \mathrm{m})\end{array}$ & $\begin{array}{c}(633- \\
<125 \mu \mathrm{m})\end{array}$ & $\begin{array}{c}(1225- \\
<250 \mu \mathrm{mm})\end{array}$ & 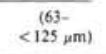 & $\begin{array}{c}(125- \\
<250 \mu \mathrm{m})\end{array}$ & 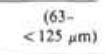 & $\begin{array}{l}(125- \\
<250 \mu \mathrm{mm})\end{array}$ & $<<125 \mathrm{pm}$ & 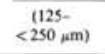 & 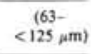 \\
\hline $\begin{array}{l}30-3,60-64 \\
30-460-64\end{array}$ & $\begin{array}{l}0.9 \\
0.1\end{array}$ & $\begin{array}{l}3.0 \\
0.2\end{array}$ & $\begin{array}{l}0.2 \\
20.1\end{array}$ & $\begin{array}{l}7.0 \\
23.5\end{array}$ & $\begin{array}{l}99.0 \\
79.0\end{array}$ & $\begin{array}{l}85.0 \\
75.0\end{array}$ & 1.0 & & & & 0.1 & 0.1 & $\begin{array}{l}0.6 \\
0.4\end{array}$ & ${ }_{0.3}^{1.9}$ & & \\
\hline $\begin{array}{l}31-1,37-39 \\
31-2,37-39 \\
31-3,37-39\end{array}$ & $\begin{array}{r}1.5 \\
12.0 \\
1.6\end{array}$ & $\begin{array}{l}3.7 \\
3.0 \\
6.0\end{array}$ & $\begin{array}{l}0.1 \\
4.8 \\
1.2\end{array}$ & $\begin{array}{r}0.1 \\
30.0 \\
4.5\end{array}$ & $\begin{array}{l}98.0 \\
78.0 \\
97.0\end{array}$ & $\begin{array}{l}95.0 \\
60.0 \\
88.0\end{array}$ & $\begin{array}{l}0.2 \\
5.0\end{array}$ & & & & & $\begin{array}{l}0.1 \\
3.0\end{array}$ & 0.1 & 0.1 & & \\
\hline $\begin{array}{l}32-1,55-57 \\
32-2,94-96 \\
\end{array}$ & $\begin{array}{r}0.9 \\
30.5\end{array}$ & $\begin{array}{l}4.1 \\
0.5\end{array}$ & $\begin{array}{r}24.0 \\
3.0\end{array}$ & $\begin{array}{r}40.0 \\
2.0 \\
\end{array}$ & $\begin{array}{l}50.0 \\
45.0\end{array}$ & $\begin{array}{l}49.0 \\
17.0\end{array}$ & $\begin{array}{r}25.0 \\
2.0\end{array}$ & 5.0 & & & & & 19,0 & 17.0 & & \\
\hline 33-1, 30-34 & 1.0 & 0.5 & 10.0 & $\begin{array}{l}28.0 \\
180\end{array}$ & $\begin{array}{l}79.0 \\
490\end{array}$ & 65.0 & $\begin{array}{r}10.0 \\
30\end{array}$ & & & & & $\begin{array}{l}0.5 \\
28.5\end{array}$ & $\cdot$ & $\cdot$ & & \\
\hline & $\begin{array}{l}2.8 \\
2.8\end{array}$ & $\begin{array}{c}11.0 \\
6.5\end{array}$ & $\begin{array}{l}45.0 \\
34.0\end{array}$ & 焉3.0 & $\begin{array}{l}49.0 \\
63.0\end{array}$ & 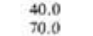 & 3.0 & & & 0.5 & & $\begin{array}{r}28.0 \\
0.1\end{array}$ & & & & \\
\hline $33-4,30-34$ & & i, & 17.0 & 8.0 & 83.0 & 90.0 & & & & & & 0.1 & 0.2 & 0.4 & & \\
\hline $\begin{array}{l}33-5,30-34 \\
33-6,30-34\end{array}$ & 0.1 & $\begin{array}{l}3.5 \\
1.9\end{array}$ & $\begin{array}{l}45.0 \\
29.0\end{array}$ & $\begin{array}{l}45.0 \\
11.0\end{array}$ & $\begin{array}{l}50.0 \\
55.0\end{array}$ & $\begin{array}{l}50.0 \\
70.0\end{array}$ & $\begin{array}{l}4.5 \\
15.0\end{array}$ & & & - & & $\begin{array}{l}1.0 \\
0.1\end{array}$ & 0.5 & 12.0 & & \\
\hline $\begin{array}{l}34-1,35-38 \\
34,-38\end{array}$ & 0.4 & 11.9. & 62.5 & 25.0 & 35.0 & 50.0 & 2.0 & & & & & 0.1 & & & & \\
\hline 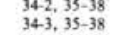 & 0.5 & $\begin{array}{l}5.5 \\
1.0\end{array}$ & $\begin{array}{l}6.8 \\
15.0\end{array}$ & 6.4 & $\begin{array}{l}85.0 \\
75.0\end{array}$ & $\begin{array}{l}60.0 \\
43.0\end{array}$ & $\begin{array}{l}0.2 \\
15.0\end{array}$ & & & & 0.1 & 0.6 & $\begin{array}{l}6.9 \\
7.9\end{array}$ & $\begin{array}{l}15.0 \\
55.0\end{array}$ & & \\
\hline $344,35-38$ & 0.8 & 2.2 & 19.0 & 37.0 & 75.0 & 60.0 & 5.0 & & & & & & 0.1 & 0.3 & & \\
\hline $35-1,30-33$ & 34.8 & 27.0 & 29.0 & 15.0 & 35.0 & 45.0 & 1.0 & & & & & 0.1 & 0.1 & 7.0 & & \\
\hline $\begin{array}{l}35-2,30-33 \\
35,-3\end{array}$ & 0.3 & 2.0 & 6.0 & 5.0 & 91.0 & 65.0 & 2.5 & & & & & 01 & 0.2 & 14.0 & & \\
\hline $\begin{array}{l}35-3,30-33 \\
35-4,30-33\end{array}$ & $\begin{array}{l}0.8 \\
20.5\end{array}$ & $\begin{array}{l}1.0 \\
2.0\end{array}$ & $\begin{array}{l}40.0 \\
17.0\end{array}$ & $\begin{array}{r}15.0 \\
4.5\end{array}$ & $\begin{array}{l}50.0 \\
55.0\end{array}$ & $\begin{array}{l}67.0 \\
80.0\end{array}$ & $\begin{array}{l}8.5 \\
1.0\end{array}$ & & & & & 0.1 & $\begin{array}{l}0.5 \\
6.5\end{array}$ & $\begin{array}{r}\begin{array}{r}15.0 \\
4.0\end{array} \\
\end{array}$ & & \\
\hline $\begin{array}{l}35-5,30-33 \\
35.6\end{array}$ & 29.5 & $\begin{array}{l}26.0 \\
310\end{array}$ & $\begin{array}{l}25,0 \\
25.0\end{array}$ & 23.0 & $\begin{array}{l}33,00 \\
450 \\
350\end{array}$ & $\begin{array}{l}80.0 \\
45.0\end{array}$ & 1.0 & & & & & & 8 & . & & \\
\hline $35-6,30-33$ & 33.0 & 33.0 & 15.0 & 10.0 & 35.0 & 40.0 & 15.0 & & & & & & 1.8 & 15.0 & & \\
\hline $36-1,28-32$ & & & 5.0 & 36.0 & 94.0 & 50.0 & & & & & - & & 0.6 & 10.0 & & \\
\hline $\begin{array}{l}36-2,30-34 \\
36-3,28-32\end{array}$ & $\begin{array}{l}0.1 \\
0.1\end{array}$ & $\begin{array}{l}5.0 \\
1.7\end{array}$ & 50.0 & 30.0 & $\begin{array}{l}48.0 \\
40.0\end{array}$ & $\begin{array}{l}{ }_{48.0}^{80.0} \\
40.0\end{array}$ & & & & & 0.1 & 0.1 & $\begin{array}{l}1.5 \\
57.5\end{array}$ & $\begin{array}{l}7.0 \\
55.0\end{array}$ & 0.1 & 0.2 \\
\hline $\begin{array}{l}36-4,28-32 \\
362\end{array}$ & 0.4 & 2.5 & & & $\begin{array}{l}40.0 \\
97.0\end{array}$ & 80.0 & & & & & 0.1 & 0.1 & 2.5 & $\begin{array}{l}33.0 \\
15.0\end{array}$ & 0.1 & \\
\hline $\begin{array}{l}37-1,30-34 \\
37-2,30-34\end{array}$ & 0.1 & $\begin{array}{l}1.5 \\
6.5\end{array}$ & $\begin{array}{r}2.0 \\
12.0\end{array}$ & $\begin{array}{l}25.0 \\
20.0\end{array}$ & $\begin{array}{l}79.0 \\
80.0\end{array}$ & $\begin{array}{l}70.0 \\
50.0\end{array}$ & 3.0 & & & $\cdot$ & & - & $\begin{array}{l}0.1 \\
4.0\end{array}$ & $\begin{array}{r}0.1 \\
20.0\end{array}$ & & \\
\hline $38-1,30-34$ & 0.4 & 1.0 & $\begin{array}{l}20.0 \\
400\end{array}$ & 30.0 & $\begin{array}{l}55.0 \\
500\end{array}$ & $\begin{array}{l}45.0 \\
\$ 50\end{array}$ & 24.0 & & & & & 0.6 & & 0.4 & & \\
\hline$\frac{38-2,30-34}{39-1,30-34}$ & 0.4 & 3.0 & 40.0 & 41.0 & 50.0 & 35.0 & 9.2 & & & & & & & & & \\
\hline $\begin{array}{l}39-1,30-34 \\
39-2,30-34\end{array}$ & 40.0 & $\begin{aligned} 40.5 \\
1.0\end{aligned}$ & 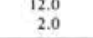 & $\begin{array}{r}18.0 \\
1.4\end{array}$ & $\begin{array}{l}35.0 \\
96.0\end{array}$ & $\begin{array}{l}40.0 \\
94,0\end{array}$ & $\begin{array}{r}12.9 \\
2.0\end{array}$ & & & & & & & & & \\
\hline $40-1,30-34$ & 50.0 & 13.0 & 1.6 & 21.0 & 32.0 & 50.0 & 6.0 & 12.5 & & - & & 0.5 & & & & \\
\hline $\begin{array}{l}40-2,30-34 \\
40-3,30-34\end{array}$ & $\begin{array}{l}4.5 \\
1.0\end{array}$ & $\begin{array}{l}7.8 \\
5.0\end{array}$ & $\begin{array}{l}1.4 \\
14.5\end{array}$ & $\begin{array}{l}7.0 \\
24.5\end{array}$ & $\begin{array}{l}91.0 \\
80.0\end{array}$ & $\begin{array}{l}85.0 \\
67.0\end{array}$ & $\begin{array}{l}3.0 \\
4.0\end{array}$ & & & & & & & & & \\
\hline $40-4,30-34$ & 9.0 & 12.0 & 11,0 & 17.0 & 55.0 & 50.0 & 25.0 & 20.0 & & - & & 0.1 & & & & \\
\hline $\begin{array}{l}40-5,30-34 \\
40-6,30-34\end{array}$ & $\begin{array}{l}11.0 \\
29.9\end{array}$ & $\begin{array}{r}5.0 \\
24.5\end{array}$ & $\begin{array}{l}5.0 \\
5.0\end{array}$ & $\begin{array}{r}2.5 \\
20.0\end{array}$ & $\begin{array}{l}80.0 \\
45.0\end{array}$ & $\begin{array}{l}90.0 \\
45.0\end{array}$ & $\begin{array}{r}4.0 \\
20.0\end{array}$ & $\begin{array}{r}2.5 \\
10.0\end{array}$ & & . & & . & & & & \\
\hline $41-1,30-34$ & 25.0 & 13.0 & & & 65.0 & 74.0 & & & & & & 0.1 & & & & \\
\hline $\begin{array}{l}{ }_{41-2,30-34}^{41-30} \\
410-34\end{array}$ & $\begin{array}{l}13.0 \\
580\end{array}$ & 5.4 & & 250 & $\begin{array}{l}60.0 \\
290\end{array}$ & $\begin{array}{l}50.0 \\
150\end{array}$ & & 50 & . & & - & 0.1 & 30 & 0.4 & & \\
\hline $\begin{array}{l}41-3,30-34 \\
41-4,34\end{array}$ & $\begin{array}{l}38.0 \\
30.0\end{array}$ & 20.0 & 2.0 & $\begin{array}{l}25,0 \\
4.0\end{array}$ & $\begin{array}{l}29.0 \\
35.0\end{array}$ & $\begin{array}{l}35.0 \\
40.0\end{array}$ & $\begin{array}{l}4.0 \\
3.0\end{array}$ & $\begin{array}{l}3.0 \\
1.0\end{array}$ & ${ }^{\circ}$ & & $\cdot$ & & 3.0 & 0.4 & & \\
\hline $\begin{array}{l}41-5,30-34 \\
41-6,30-34\end{array}$ & $\begin{aligned} 30.0 \\
6.0\end{aligned}$ & $\begin{array}{l}5.5 \\
3.1\end{array}$ & $\begin{array}{l}2.0 \\
1.5\end{array}$ & & $\begin{array}{l}38.0 \\
65.0\end{array}$ & $\begin{array}{l}44.0 \\
35.0\end{array}$ & 3.0 & & & & & & 8.0 & & & \\
\hline $42-1,30-34$ & 40.0 & 25.0 & & & 42.0 & 45.0 & & & & & & & 1.0 & & & \\
\hline 42-2, $30-34$ & 40.2 & 45.4 & & & 15.5 & 9.5 & & & & & & & & (3, & & \\
\hline $\begin{array}{l}42-3,30-34 \\
42-430-34\end{array}$ & $\begin{array}{l}78.0 \\
9.5\end{array}$ & 61.4 & 0.5 & 0.5 & 1.0 & 2.0 & & & & & & 0.1 & & & & \\
\hline $\begin{array}{l}42-4,50-34 \\
42 \cdot 5,30-34\end{array}$ & 10.0 & 88.0 & & & 50.0 & 12.0 & & & & & & 0.1 & 0.9 & & . & \\
\hline $43-1,30-34$ & 70.0 & 77.0 & & & 27.0 & 20.0 & & & & & & & 0.1 & 0.1 & & \\
\hline $44-1,30-34$ & 75.0 & 30.0 & & & 14.0 & 14.0 & & & & & & 0.1 & & & & \\
\hline $44-2,30-34$ & 85.0 & 40.0 & & & & 10.0 & & & & & & 0.1 & 0.5 & 0.1 & & \\
\hline 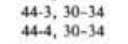 & $\begin{array}{l}40.0 \\
34.0\end{array}$ & $\begin{array}{l}34.0 \\
24.0\end{array}$ & & & $\begin{array}{l}\begin{array}{l}45.0 \\
65.0\end{array}\end{array}$ & $\begin{array}{l}60.0 \\
60.0\end{array}$ & & & & & & 0.1 & & & & \\
\hline $45-1,30-34$ & 50.0 & 60.0 & & & 48.0 & 35.0 & & & & & & 0.1 & 0.2 & $\dot{1}$ & & \\
\hline $45-2,30-34$ & 99.0 & 95.0 & & & 0.7 & 8.0 & - & & & & & 0.1 & 0.2 & 0.1 & & \\
\hline 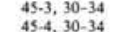 & $\begin{array}{l}50.0 \\
480\end{array}$ & $\begin{array}{l}75.0 \\
150\end{array}$ & & & $\begin{array}{l}45.0 \\
50.0\end{array}$ & $\begin{array}{l}20.0 \\
490\end{array}$ & & & & & & 0.1 & 0.3 & 0.1 & & \\
\hline 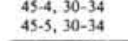 & & 15.0 & & & $\begin{array}{l}50.0 \\
49.0\end{array}$ & $\begin{array}{l}49.0 \\
49.0\end{array}$ & 1.5 & & & & & & & & & \\
\hline $\begin{array}{l}46-1,30-34 \\
46-2,30-34\end{array}$ & $\begin{array}{l}85.0 \\
997\end{array}$ & $\begin{array}{l}83.5 \\
998\end{array}$ & 0.1 & 0.1 & $\begin{array}{r}14.0 \\
0.1\end{array}$ & 15.0 & 0.3 & & & & 0.2 & 1.0 & & & & \\
\hline $\begin{array}{l}46-3,30-34 \\
46-4,30-34\end{array}$ & $\begin{array}{l}100.0 \\
075\end{array}$ & 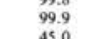 & 0.1 & & & $\begin{array}{l}0.1 \\
0.1 \\
4.0\end{array}$ & & & & & 0.5 & 450 & & & & \\
\hline$\frac{46-4,30-34}{48-1,42-44}$ & $\begin{array}{l}9.3 \\
55.0\end{array}$ & $\begin{array}{l}45.0 \\
80.0\end{array}$ & 20 & & $\frac{0.5}{33.0}$ & $\begin{array}{l}4.0 \\
9.0\end{array}$ & 25 & & & & & 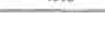 & & & & \\
\hline $49-1,30-34$ & 100.0 & 100,0 & & & & & & & & & & & & & & \\
\hline 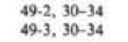 & $\begin{array}{l}99.9 \\
99.9\end{array}$ & $\begin{array}{l}99.9 \\
99.9\end{array}$ & & & $\begin{array}{l}0.1 \\
0.1\end{array}$ & $\begin{array}{l}0.1 \\
0.1\end{array}$ & & & & & & & & & & \\
\hline $50-1,30-34$ & 99.9 & 99.9 & & & 0.1 & 0.1 & & & & & & & & & & \\
\hline $50-2,30-34$ & 99.9 & 99.9 & & & 0.1 & 0.1 & & & & & & & & & & \\
\hline 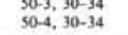 & $\begin{array}{l}1000.0 \\
100.0\end{array}$ & $\begin{array}{l}1000.0 \\
100.0\end{array}$ & & & & & & & & & & & & & & \\
\hline $\begin{array}{l}51-1,30-34 \\
51 \cdot 2,30-34\end{array}$ & $\begin{array}{l}1000.0 \\
100.0\end{array}$ & $\begin{array}{l}100.0 \\
100.0\end{array}$ & & & & & & & & & & & & & & \\
\hline & 99.9 & 99.9 & & & 0.1 & 0.1 & & & & & & & & & & \\
\hline $52-2,30-34$ & $\begin{array}{l}99.9 \\
095\end{array}$ & 90.9. & & & 0.1 & 0.1 & & & & & & & & & & \\
\hline$\$ 2-4,30-34$ & $\begin{array}{l}99.9 \\
99.9\end{array}$ & $\begin{array}{l}94.3 \\
999\end{array}$ & & & 0.9 & 0.5 & & & & & & & & & & \\
\hline $52 \cdot 5,30-34$ & 99.9 & 99.9 & & & $\begin{array}{l}0.1 \\
0.1\end{array}$ & $\begin{array}{l}0.1 \\
0.1\end{array}$ & & & & & & & & & & \\
\hline $\begin{array}{l}53-1,30-34 \\
53-2,2,30-34\end{array}$ & $\begin{array}{r}100.0 \\
99.9\end{array}$ & $\begin{array}{r}100,0 \\
99.5\end{array}$ & & & 0.1 & 0.4 & & & & & & & & & & \\
\hline $54-1,30-34$ & 38.5 & 22.0 & & & 60.0 & 55.0 & 0.2 & & & 0.1 & 1.0 & 18.0 & 0.1 & 0.1 & & \\
\hline
\end{tabular}


Table 3 (continued).

\begin{tabular}{|c|c|c|c|c|c|c|c|c|c|c|c|c|c|c|}
\hline \multicolumn{15}{|c|}{ Fossils } \\
\hline \multicolumn{4}{|c|}{ Calcarroous } & \multicolumn{6}{|c|}{ Siliceous } & \multicolumn{5}{|c|}{ Other } \\
\hline \multicolumn{2}{|c|}{ 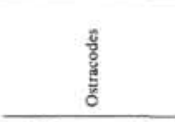 } & \multicolumn{2}{|c|}{ t气 } & \multicolumn{2}{|c|}{ 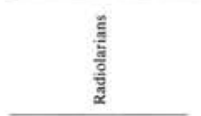 } & \multicolumn{2}{|c|}{ 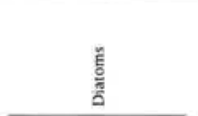 } & \multicolumn{2}{|c|}{ 䇾总 } & \multicolumn{2}{|c|}{ 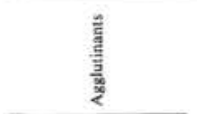 } & \multicolumn{2}{|c|}{ 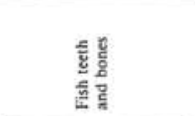 } & 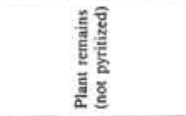 \\
\hline \multirow[t]{33}{*}{$\begin{array}{c}(125- \\
<250 \mu \mathrm{m}) \\
\end{array}$} & $\begin{aligned} & 163- \\
&<125 \mu \mathrm{rr}\end{aligned}$ & $\begin{array}{l}<125- \\
<250 \mu \mathrm{m})\end{array}$ & $\begin{array}{l}\frac{(63-}{<125 \mu \mathrm{m})} \\
<-10\end{array}$ & $\begin{array}{l}\begin{array}{c}(125- \\
<250 \mu \mathrm{m})\end{array} \\
\end{array}$ & $\begin{array}{l}<125 \mu \mathrm{mm}) \\
<\frac{(63-}{10}\end{array}$ & $\begin{array}{l}(125- \\
<250 \mu \mathrm{m})\end{array}$ & $\begin{array}{l}\frac{(63-}{<125 \mu \mathrm{m})} \\
\end{array}$ & 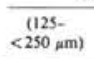 & 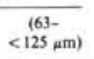 & 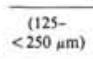 & $\frac{(633-}{<125 \mu \mathrm{m})}$ & $\begin{array}{l}<250 \mu \mathrm{m}) \\
<250\end{array}$ & $\begin{array}{l}\frac{(63-}{<125 \mu \mathrm{m})} \\
\end{array}$ & $\begin{array}{ll}(125- & (63- \\
<250 \mu \mathrm{m}) & <125 \mu \mathrm{m})\end{array}$ \\
\hline & & & & & & & & & & & & $\begin{array}{l}0.2 \\
0.4\end{array}$ & $\begin{array}{l}2.0 \\
1.0\end{array}$ & \\
\hline & & & & & & & & & & & & $\begin{array}{l}0.1 \\
0.2 \\
0.2\end{array}$ & $\begin{array}{l}1.0 \\
4.0 \\
1.5\end{array}$ & \\
\hline & & & & & & & & & & & & $\begin{array}{l}0.1 \\
0.5\end{array}$ & $\begin{array}{l}1.9 \\
2.5\end{array}$ & \\
\hline & & & & & 0.1 & & & & & 0.1 & & $\dot{0.1}$ & $\begin{array}{l}3.0 \\
2.5 \\
0.4\end{array}$ & \\
\hline & & & & & & & & & & . & & $\begin{array}{l}0.1 \\
0.4 \\
0.5\end{array}$ & $\begin{array}{l}1.0 \\
5.0\end{array}$ & \\
\hline & & & & & & & & & & $\begin{array}{l}0.1 \\
0.1\end{array}$ & & $\begin{array}{l}0.4 \\
0.5 \\
0.9 \\
0.1 \\
\end{array}$ & $\begin{array}{r}13.0 \\
3.0 \\
1.0 \\
0.5\end{array}$ & \\
\hline & & & & & & & & & & & & 0.1 & $\begin{array}{r}5.9 \\
14.0\end{array}$ & \\
\hline & & & & & & & & & & & & 0.2 & 2.9 & \\
\hline & & & & & & & & & & & & $\begin{array}{l}0.5 \\
0.2\end{array}$ & $\begin{array}{l}5.5 \\
2.0\end{array}$ & \\
\hline & & & & & & & & & & 2.6 & & $\begin{array}{l}0.4 \\
0.4 \\
0.5 \\
0.1\end{array}$ & $\begin{array}{r}4.0 \\
10.0 \\
3.0 \\
2.5\end{array}$ & \\
\hline & & 0.5 & 0.5 & & & & & & & & & $\begin{array}{l}0.8 \\
0.5\end{array}$ & $\begin{array}{l}3.4 \\
3.0\end{array}$ & \\
\hline & & & & & & & & & & & & $\begin{array}{l}1.0 \\
0.1 \\
\end{array}$ & $\begin{array}{r}23.0 \\
1.0\end{array}$ & \\
\hline & & & & & & & & & & & & 0.1 & $\begin{array}{l}1.5 \\
1.5\end{array}$ & \\
\hline & & & & & & & & & & & & $\begin{array}{l}0.4 \\
0.1 \\
0.5\end{array}$ & $\begin{array}{l}3.0 \\
0.2 \\
3.5 \\
0.9\end{array}$ & \\
\hline & & & & & & & & & & & & 0.1 & 0.5 & \\
\hline & & & & & & & & & & $\begin{array}{r}9.5 \\
38.5\end{array}$ & 12.0 & 0.5 & 0.9 & \\
\hline & & & & $\begin{array}{l}3.0 \\
50\end{array}$ & $\begin{array}{r}28.0 \\
100\end{array}$ & & & - & 0.1 & 30 & & & 1.0 & \\
\hline & & & & $\begin{array}{l}5.0 \\
0.4\end{array}$ & $\begin{array}{r}25.0 \\
2.0\end{array}$ & & & & & $\begin{array}{l}25.0 \\
25.0 \\
160\end{array}$ & $\begin{array}{l}25.3 \\
25.0 \\
40.0\end{array}$ & 0.1 & $\begin{array}{l}0.5 \\
0.5 \\
1.9\end{array}$ & \\
\hline & & & & 1.9 & 10.0 & & & 0.1 & & 15.0 & 17.9 & & 0.1 & \\
\hline & & & & $\begin{array}{r}4.0 \\
15.0\end{array}$ & $\begin{array}{l}35.0 \\
150\end{array}$ & & & & & $\begin{array}{l}40.0 \\
5.0\end{array}$ & $\begin{array}{l}{ }_{10.0}^{10.0} \\
200\end{array}$ & $\begin{array}{l}0.3 \\
0.5\end{array}$ & 1.0 & \\
\hline & & & & 0.2 & 2.0 & & & & 0.1 & 0.3 & 1.0 & 0.1 & 0.2 & \\
\hline & & & & 14.0 & 0.1 & & & & • & 25.0 & 1.9 & 0.1 & & \\
\hline & & & & 1.0 & 2.5 & & & & 0.1 & 1.8 & 0.1 & 0.1 & 0.2 & \\
\hline & & & & $\begin{array}{r}10.5 \\
5.0\end{array}$ & $\begin{array}{l}55,0 \\
35.0\end{array}$ & & & & & $\begin{array}{l}0.1 \\
9.3\end{array}$ & & 0.4 & $\begin{array}{l}0.9 \\
0.3\end{array}$ & \\
\hline & & & & $\begin{array}{l}3.0 \\
10.0\end{array}$ & $\$ 5.0$ & & & & & 5.0 & 9.8 & & 0.1 & \\
\hline & & & & 0.5 & 15.5 & & & & 0.1 & & & 0.5 & 0.4 & \\
\hline & & & & 0.1 & $\begin{array}{l}4.8 \\
0.9\end{array}$ & & & & & L.S & 1.0 & 0.2 & & \\
\hline & & & & 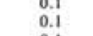 & $\begin{array}{l}0.5 \\
0.5\end{array}$ & & & & 0.1 & 4.5 & 4.0 & 0.1 & 0.2 & \\
\hline & & & & $\begin{array}{l}0.1 \\
6.9\end{array}$ & $\begin{array}{l}35.0 \\
35.0\end{array}$ & & & & & $\begin{array}{r}0.4 \\
44.0\end{array}$ & $\begin{array}{r}0.9 \\
16.0\end{array}$ & 0.1 & 0.1 & \\
\hline & & & & 0.3 & 0.4 & & & & & 0.1 & & 0.1 & • & \\
\hline & & & & 1.5 & 6.0 & & & & & & & & & \\
\hline & & & & 0.5 & 0.9 & & & & & 7.0 & 10.0 & 0.1 & & \\
\hline
\end{tabular}




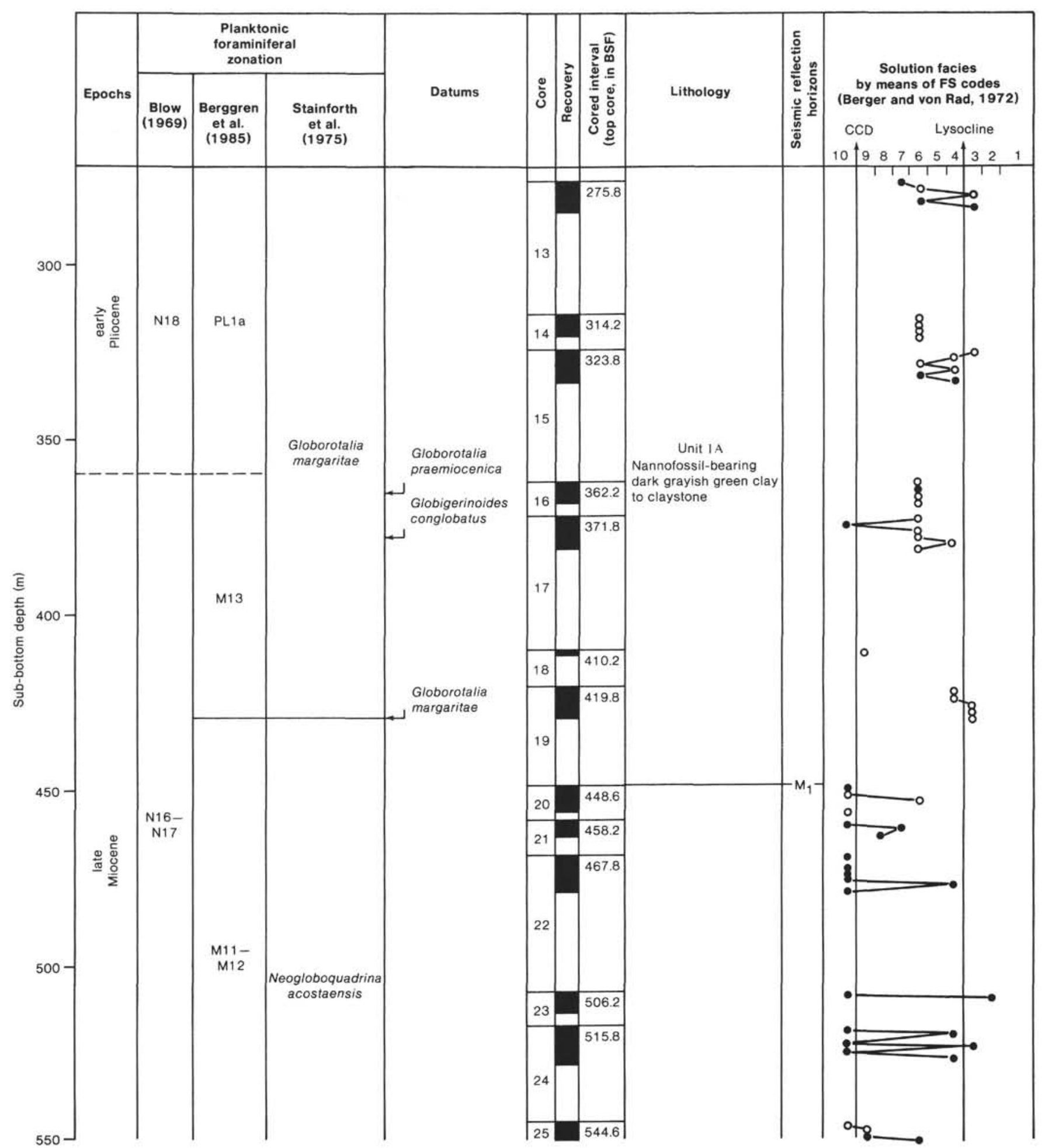

Figure 2. Diagram of the solution facies of samples from Site 603, Hole 603 of Leg 93, showing foraminiferal dissolution codes (FS codes). The foraminiferal code values (FS values) represent 10 states of preservation of calcareous foraminifers, given by Berger and von Rad (1972). Washed residues of reworked sediments contain $\geq 3 \%$ quartz, Quinqueloculina sp., reworked Cretaceous foraminifers, $\geq 95 \%$ rock fragments (predominantly silt), echinoid, ostracode, and shell fragments, and glauconite, or a combination of these components. Exact location of $\mathrm{M}_{2}$ uncertain because of sampling gap. 


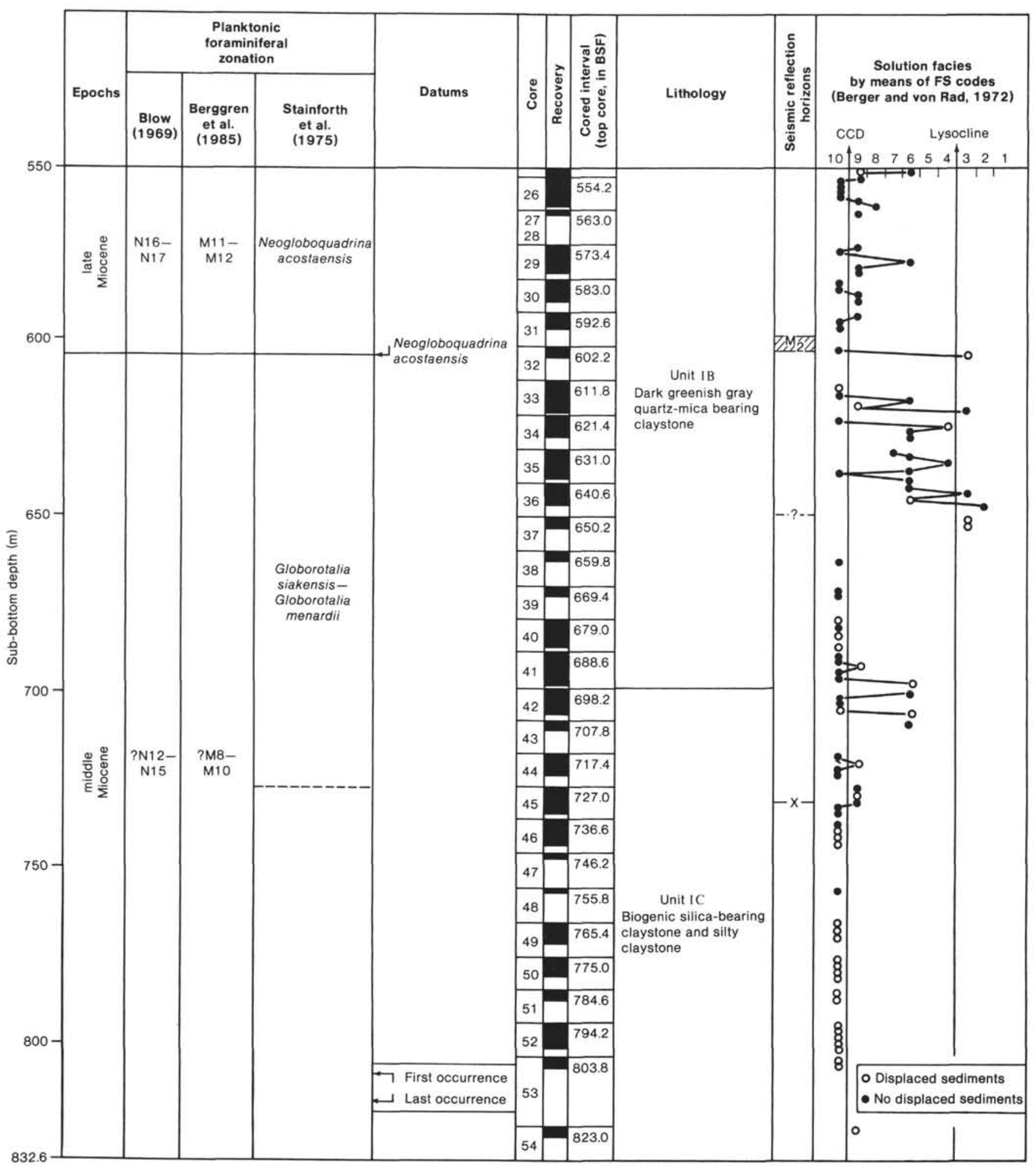

Figure 2 (continued). 
Table 4. Solution susceptibility ranking of planktonic foraminifers including rank factors from delicate (1) to resistant (8), after Hemleben and Auras (1984).

\begin{tabular}{|c|c|c|c|}
\hline $\begin{array}{l}\text { Sediment ranking } \\
\text { (this chapter) }\end{array}$ & $\begin{array}{l}\text { Rank } \\
\text { factor }\end{array}$ & $\begin{array}{l}\text { Neogene } \\
\text { species }\end{array}$ & $\begin{array}{l}\text { Rank } \\
\text { factor }\end{array}$ \\
\hline Hastigerina pelagica & 1 & & \\
\hline Globigerina falconensis & 2 & & \\
\hline Globigerinella aequilateralis & 3 & Globorotalia praehirsuta & 4 \\
\hline Globigerinoides ruber & 3 & G. margaritae & 4 \\
\hline G. sacculifer & 4 & G. miocenica & 4 \\
\hline Orbulina universa & 4 & G. multicamerata & 4 \\
\hline Globigerinoides conglobatus & 5 & & \\
\hline Globorotalia inflata & 5 & Globigerinoides altispira & 5 \\
\hline G. menardii & 5 & Globorotalia tosaensis & 6 \\
\hline G. truncatulinoides & 6 & Neogloboquadrina humerosa & 6 \\
\hline Neogloboquadrina dutertrei & 6 & Neogloboquadrina acostaensis & 6 \\
\hline G. crassaformis & 6 & & \\
\hline Pulleniatina obliquiloculata & 7 & Globigerina nepenthes & 7 \\
\hline G. tumida & 7 & & \\
\hline N. pachyderma & 7 & & \\
\hline Sphaeroidinellopsis dehiscens & 8 & Sphaeroidinellopsis paenedehiscens & 8 \\
\hline
\end{tabular}

Note: Resistant forms $=$ species with a solution susceptibility $\geq 5$ (this chapter). 